\title{
The Lunar Problem is The Barrier of The Future Time of The Earth
}

\author{
Eduardo S. Guimaraes
}

Intellectual, Writer, and Inventor

edu.guimaraes8@gmail.com

\section{Abstract}

1. This article "The lunar problem is the barrier of the future time of the Earth" is a logical and rational analysis of the formation of the nuclear universe with galaxies, stars, the Sun star, the system of planets and the moons, and arrives at new original and inedited conclusions.

2. The Big Bang of the primitive universe is a sequence programmed by the nature of thermonuclear super explosions in sidereal space.

3. These thermonuclear super explosions swept nuclear sidereal space generating the large mass islands of galaxies like the Milky Way.

4. The Milky Way was the first generation of the hyper-bubbles of the mixture of nuclear masses, which are: geological nuclear mass of attraction of gravity; geological nuclear mass of orbital attraction; geological nuclear mass of orbital repulsion.

5. Because of nuclear hyper tremors, the nucleus of the Milky Way generated the second generation of the super bubbles that were repulsed from the galaxy's nucleus by the action of the geological nuclear mass of orbital repulsion, and then the super bubbles became in the many billions of celestial stars that make up the galactic disc.

6. Because of the hyper tremors, the nucleus of these billions of stars, including the Sun, generated the third generation of super bubbles and large bubbles that were repulsed from the nuclei of the stars by the action of the geological nuclear mass of orbital repulsion, and then the super bubbles and large bubbles have become the sequence of planets, which makes up the orbital disk of the solar system.

7. Due to a small number of nuclear super tremors, the nucleus of the sequence of planets tremble, shook and generated the fourth generation of the small bubbles that were repulsed from the nucleus of the planetary sequence by the action of the geological nuclear mass of orbital repulsion, and then, the small bubbles have become the sequence of moons, which makes up the orbital disk of the planets.

8. And so, began the count the new time, after the sequence of Big Bang explosions.

Date of Submission: 27 September, 2018

DOI: 10.24297/jap.v14i3.7802

ISSN: $2347-3487$

Volume: 14 Issue: 3

Journal: Journal of Advances in Physics

Website: https://cirworld.com

This work is licensed under a Creative Commons Attribution 4.0 International License. 


\section{Keywords}

Time, Big Bang, Nucleus, Solar System, Galaxy, Cosmos, Universe, Orbital Superconductivity, Magnetism, Gravity, Space, Orbit, Super moon, Sun, Mercury, Venus, Earth, Moon, Mars, Ceres, Jupiter, Saturn, Neptune, Uranus, Pluto, Space Energy, Nuclear Energy, Galactic Disk, Asteroid Belt, Saturn Rings, Milky Way.

\section{Academic Discipline}

Physics, Geology, Geometry, Astronomy, Magnetism, Nuclear Energy

\section{Subject Classification}

Physics

\section{Introduction}

"The lunar problem is the barrier of the future time of the Earth" is a profound study in the disciplines of physics, astronomy, geology, geometry, orbit, gravity, Earth magnetism, nucleus nuclear energy, which explains the existence of the physical phenomenon of fall of the moon by the loss of level of the geological energy of orbital repulsion of the lunar nucleus.

Eduardo Santos Guimarães in the study "Theory of the three fields of space", published in 2018 by JOURNAL OF ADVANCES IN PHYSICS, explains the existence of the three fields of space: Gravity Field, Field of Magnetism and Orbital Field.

Based on this study, Eduardo Santos Guimarães presents the study "The lunar problem is the barrier of the future time of the Earth", that explains the natural origin of the moon by the motivation of a nuclear shake and tremble of the big bang, that produced a deformation of the mass by the formation of a bubble within the Earth's core at a time when the mass of the planet Earth was in an incandescent liquid state and was moving in the orbit of the planet.

The study "The lunar problem is the barrier of the future time of the Earth", also explains the loss of the nuclear energy of the moon based on the analysis of the dimensional differential of the nuclear masses between the Earth and the Moon.

The study "The lunar problem is the barrier of the future time of the Earth", explains the aging of the moon in the loss of kinetic energy of the rotation movement in the lunar axis, caused by the loss of thermogravitational energy of the lunar nucleus, which produces the stop of the rotation of the moon by the solidification of the lunar nucleus.

The study "The lunar problem is the barrier of the future time of the Earth", finally explains the aging of the moon in the loss of energy level of the lunar orbital field (produces the distance Earth, Moon) caused by the loss of thermo-orbital repulsion energy of the lunar nucleus, which produces the fall of the moon, and generates the decrease of the area of the lunar orbital ellipse, and in turn, generates the disaster of the collision.

\section{The geological origin of gravity on the moon}

The gravity originates from the nuclear energy generated by the geological nuclear mass of gravity attraction that is present in the geology of the moon. The geological nuclear mass of gravity attraction when in liquid state makes the rotational of the moon's nucleus.

\section{At the nucleus of the moon, the energy is naturally transformed into a field of lunar gravity}

The thermo-gravitational energy is naturally transformed into a field of gravity of the moon. The gravitational field of the moon is not observed in astronomy because the moon does not have a rotation movement with 360 degrees. By the moon's movement of revolution on the Earth, we know that the moon 
has a small field of gravity.

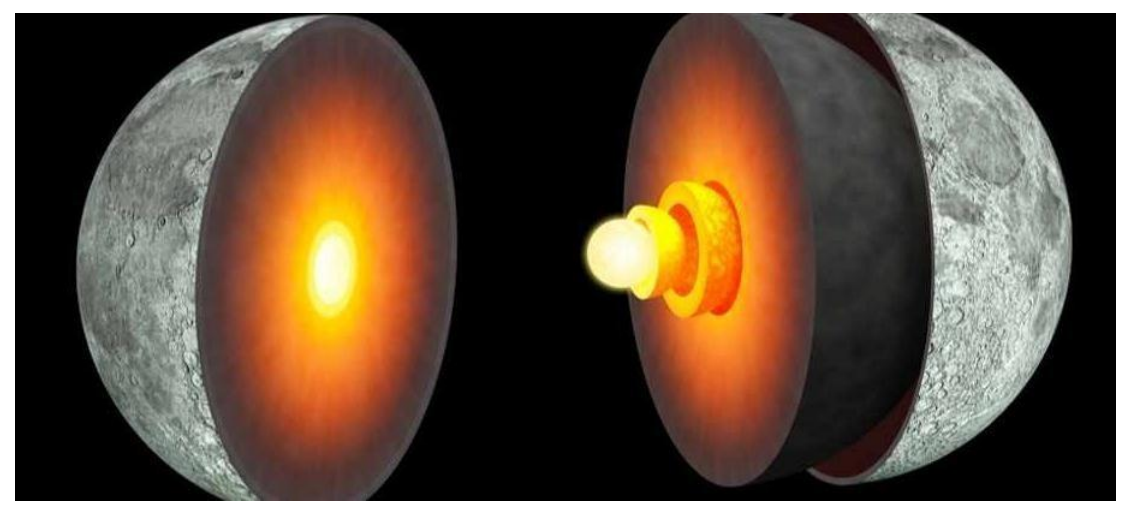

The gravity is visible on the moon because it is a mass attraction phenomenon. The phenomenon of gravity is visible in the man's walk on the moon.

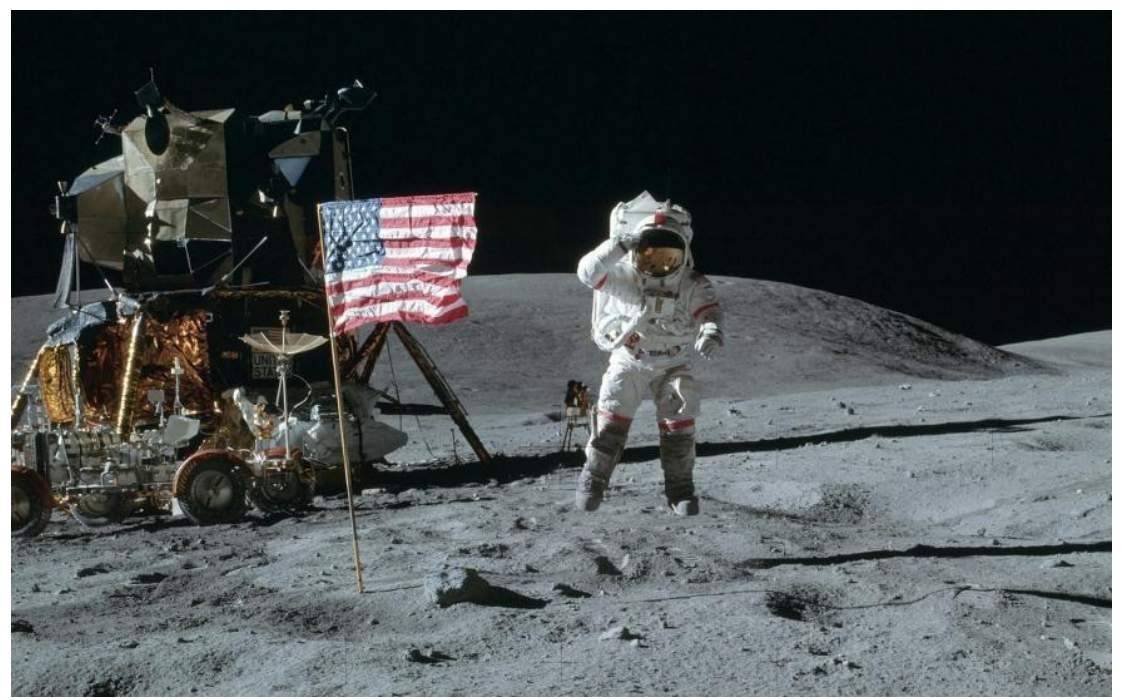

\section{Superconductivity of gravity}

On July 5, 1687, Sir Isaac Newton published a three-volume work known as Philosophiae Naturalis Principia Mathematica. In the first edition of this work, Isaac Newton reveals the discovery of gravity through the Theory of Universal Gravitation. However, Sir Isaac Newton could not explain the origin of gravity, and then, considered gravity as a force. But in fact, gravity is a superconductivity of attraction of gravity, produced in the nuclear geology of the Earth's core and also in the other celestial bodies. The superconductivity of gravity is generated by the geological nuclear mass of gravity attraction, which produces a gravitational field that attracts all matter with certain force to the center of the moon.

\section{The gravity field of the moon}

The thermo-gravitational energy of the moon generates the field of gravity that is divided into zones with microgravity; spatial gravity; surface gravity; underground gravity; and nucleus gravity. 


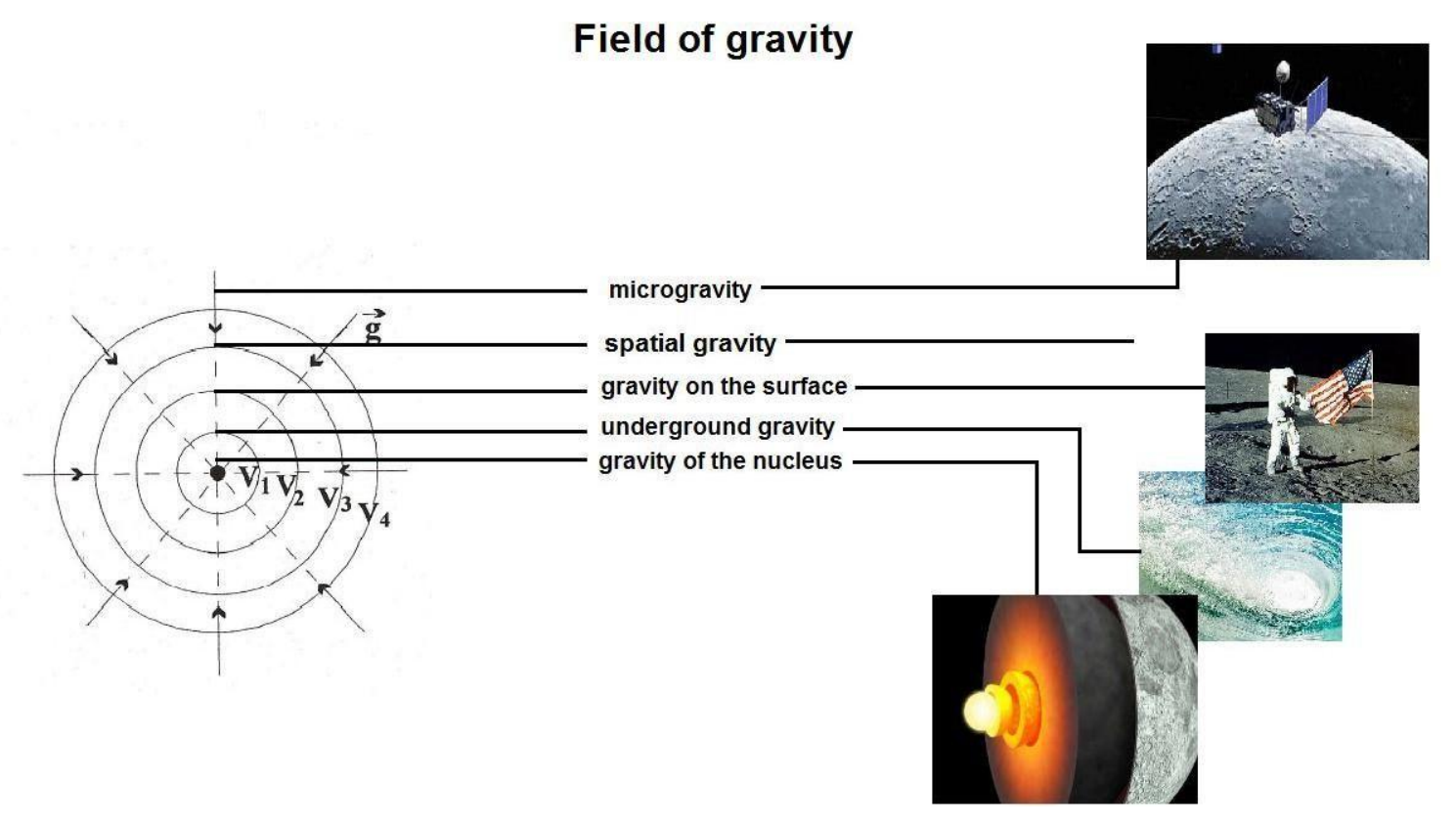

\section{The microgravity of the moon}

The microgravity of the moon produces a small geostationary orbital zone. Above this orbital zone, the lunar satellite changes from lunar orbit to space. Below this orbital zone, the satellite falls on the surface of the moon.

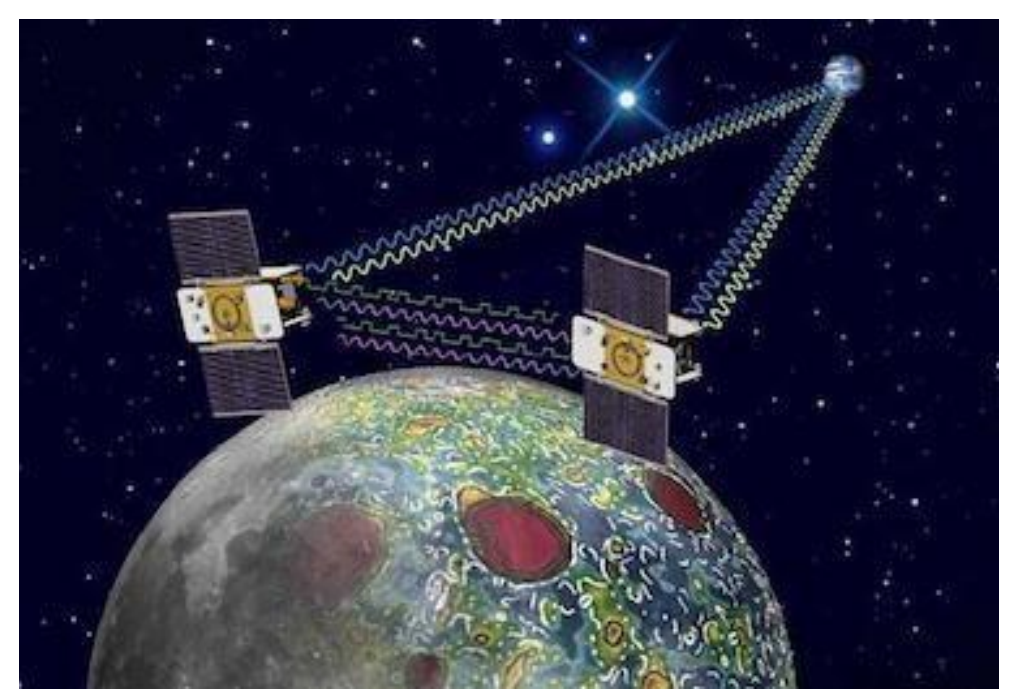

\section{The space gravity field of the moon}

The space gravity field of the moon is the gravity field of space, above the lunar surface. The gravity of the lunar space

$<1.62 \mathrm{~m} / \mathrm{s}^{2}$. 
The gravity on the surface of the moon

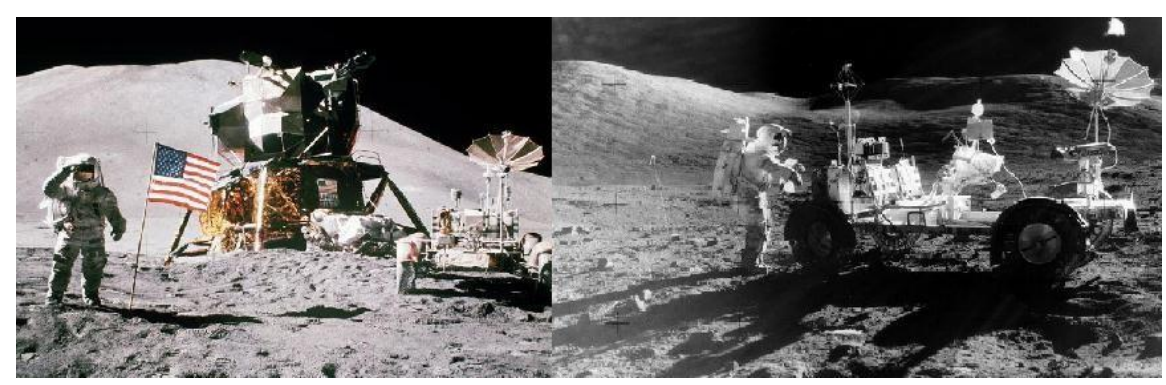

The gravity on the lunar surface is the zone of the lunar surface that the man made the walk on the moon. The gravity on the lunar surface $=1.62 \mathrm{~m} / \mathrm{s}^{2}$.

\section{The underground gravity within the moon rock}

The lunar underground gravity is the zone of flow of lunar water volumes. The lunar underground gravity > $1.62 \mathrm{~m} / \mathrm{s}^{2}$.

\section{The gravity inside the moon's nucleus}

The gravity within the lunar nucleus is the natural generation zone of lunar gravity. The nucleus of the moon is solidified, so the moon does not have a 360 -degree rotational motion on its lunar axis. The gravity of the lunar nucleus is much greater than $1.62 \mathrm{~m} / \mathrm{s}^{2}$.

\section{The geometric curve of gravity}

The parabola is the gravity curve that is visible through the mass curve.

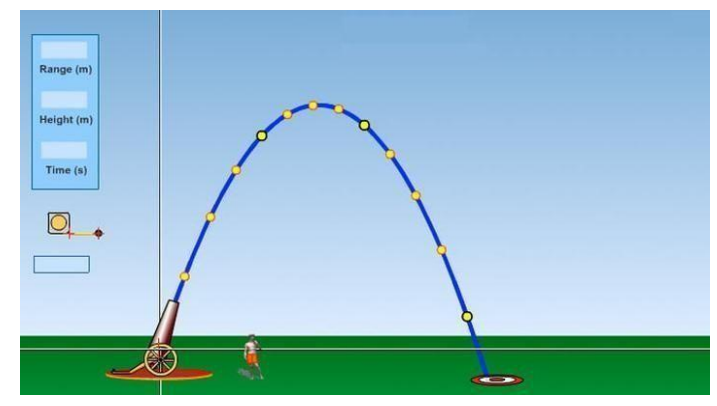

\section{No gravity in space}

In space there is no gravity. 


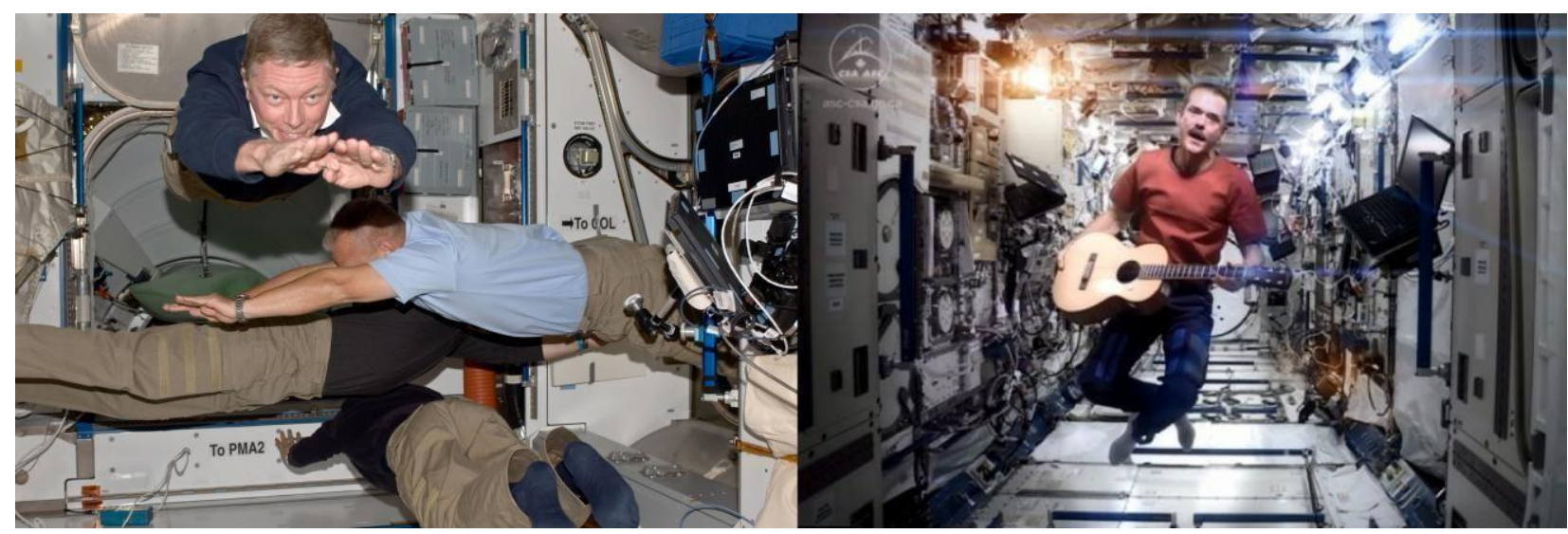

\section{The geological origin of the magnetic field of the moon}

The moon's magnetism originates from the nuclear energy generated by the geological nuclear mass of orbital attraction that is present in the geology of the moon.

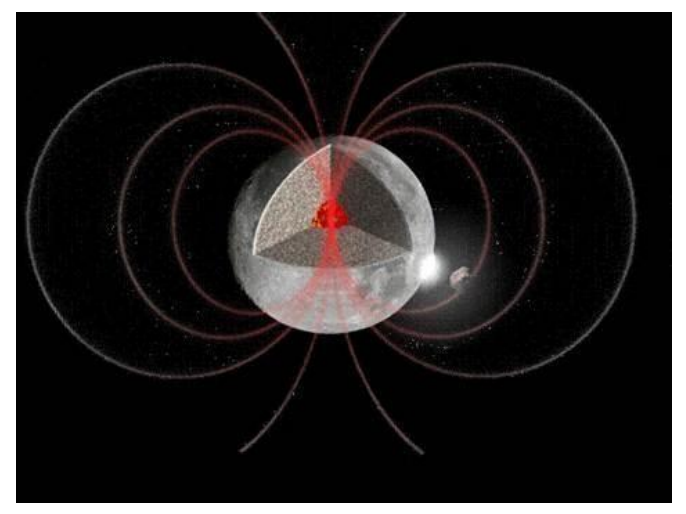

\section{On the moon, energy is naturally transformed into a nuclear magnetic field}

Thermo-magnetic energy is naturally transformed into a magnetic field of the moon. The lunar magnetism is observed by the illustration of the nucleus of the moon. The nucleus of the moon is a thermo-nuclear superconductor.

\section{Nuclear magnetism}

In 1600, William Gilbert published the work "Of Magnete, Magneticisque Corporibus and Magno Magnete Tellure", which reveals the discoveries about the great magnet of the Earth. Then, William Gilbert, revealed the origin of nuclear magnetism, produced in the nuclear geology of the Earth and the moon. Eduardo Guimarães later explained that the moon's nucleus was not made of iron and nickel but was made of a solid nuclear mass that causes nuclear magnetism not to be destroyed by the high temperature of the lunar nucleus, well above Curie point. This solid nuclear mass creates the lunar phenomena of the moon's south magnetic pole, and the spatial phenomenon of the moon's nuclear magnetic field. 


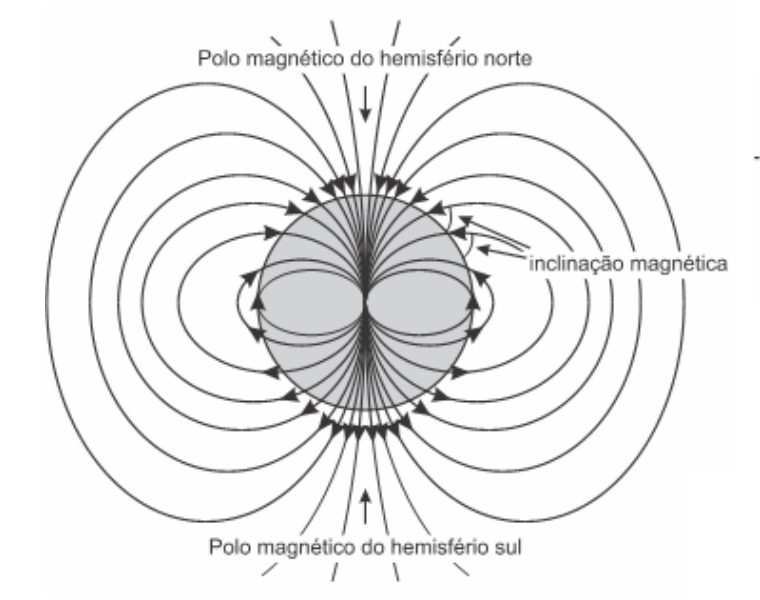

The nuclear magnetic field of the moon

The nuclear magnetic field of the moon is weak, and has no property able to bend the light.

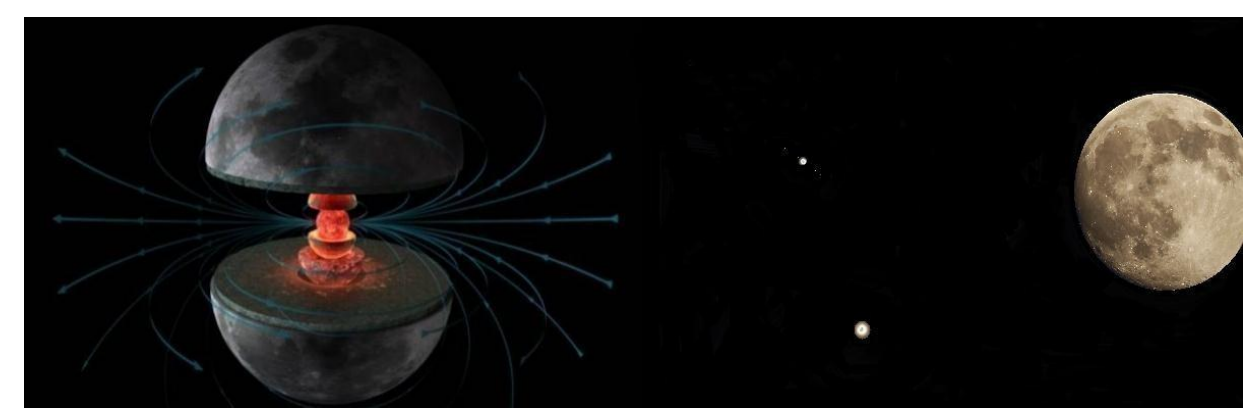

\section{The nuclear micro-magnetism of the moon}

The micro-compass points to the incidence of nuclear micro magnetism of the lunar nuclear magnetic field.

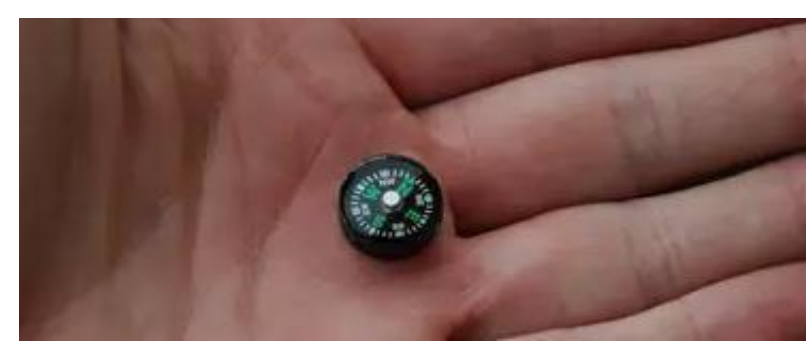




\section{The magnetism on the surface of the moon}

The magnetism on the surface of the moon is identified by the micro-compass.

\section{The Moon's underground magnetism}

The underground magnetism inside the moon is stronger.

The geometric curve of magnetism

The geometric curve of magnetism is an invisible nephroid curve extending between the two north and south poles.
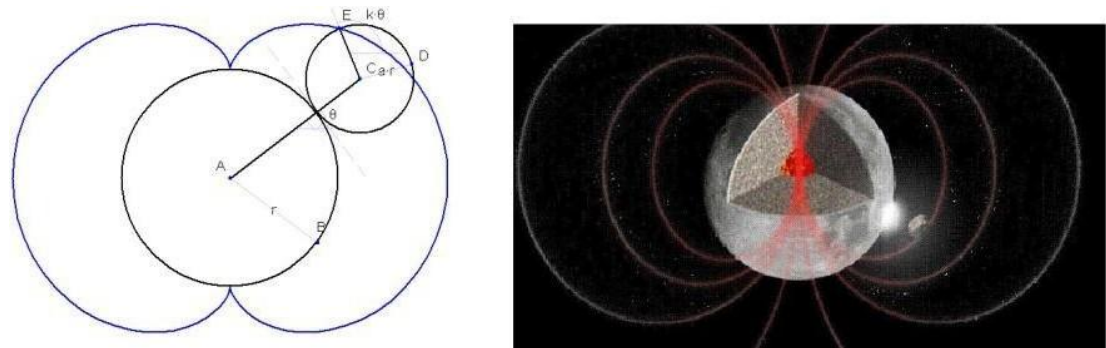

\section{The nuclear magnetic field in the space between the Earth and the moon}

By the nuclear magnetic field of the nucleus of planet Earth in connection with the moon, we can establish a spatial and computerized communication between the Earth and the lunar mission. For this it is necessary to develop the nuclear magnetic superconductor of telecommunication in the space distance.

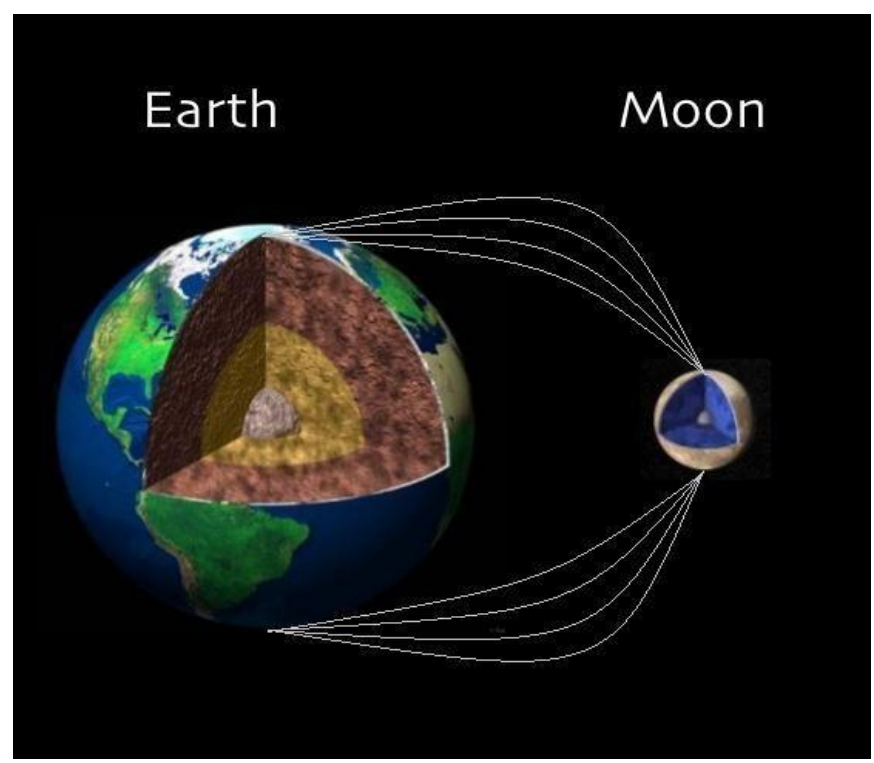




\section{The geological origin of the moon's orbit}

The lunar orbit originates from the nuclear energy generated by the mixing of the geological nuclear mass of orbital attraction with the geological nuclear mass of orbital repulsion, which is found in the geology of the moon's nucleus. The mixture of these two nuclear masses creates the distance and elliptical orbital curvature of the moon.

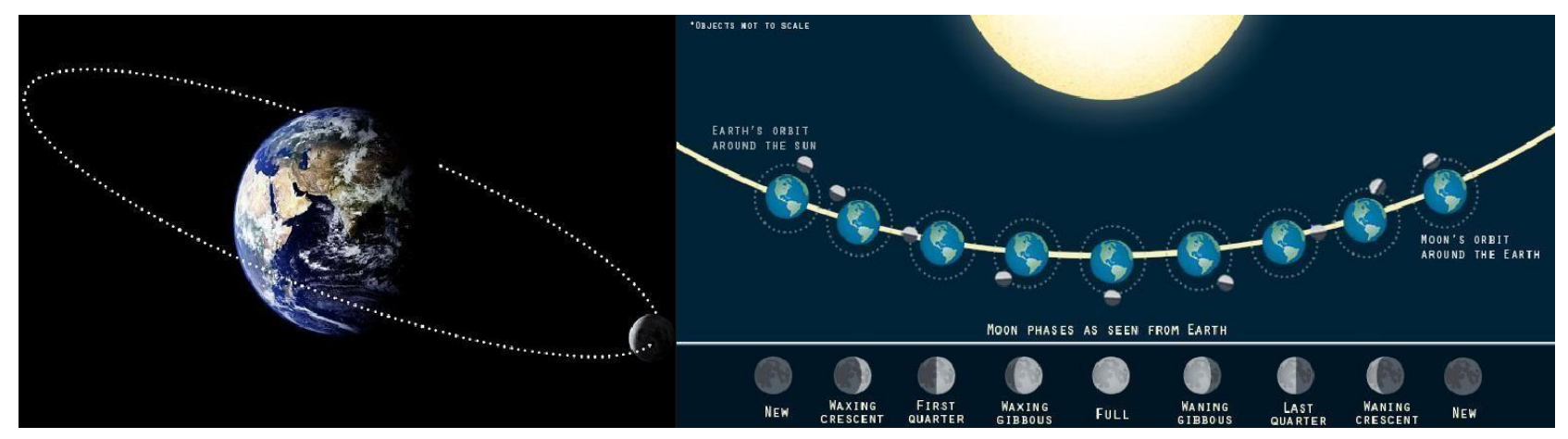

\section{In the nucleus of the moon, the energy is naturally transformed into orbital field}

The subtraction of the geological energy of thermo-orbital repulsion with the geological energy of thermoorbital attraction is naturally transformed at the lunar moment by the orbital field between the Earth and the moon. The orbital field of the moon is invisible in astronomy, but is noticeable by the moon's movement of revolution. By the orbiting revolution of the moon, we know that the moon has a small orbital field that creates the orbital distance between the planet Earth and the moon.

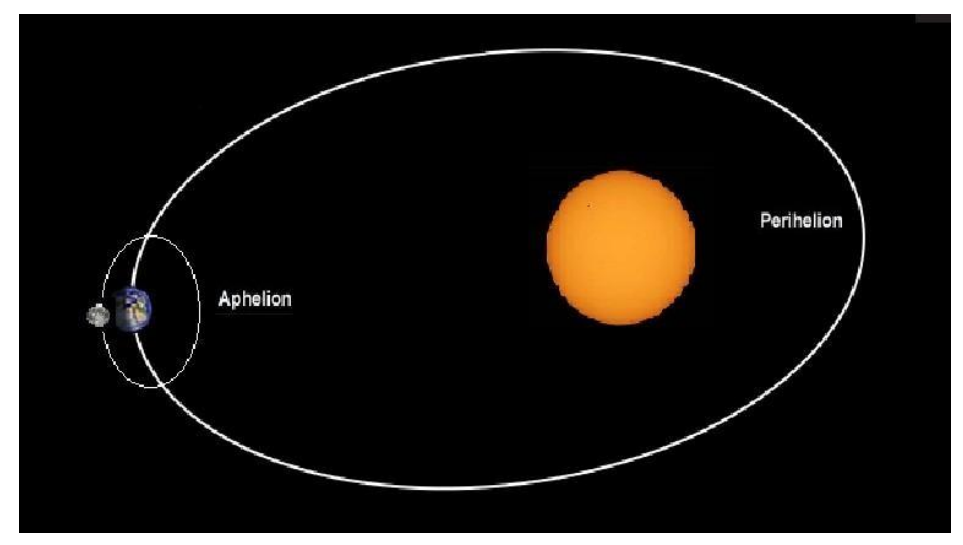

Due to the existence of the movement of the seas, we know that the orbital field of the moon displaces the planet Earth, whenever the rotation of the Earth on the axis passes through the moon, and the space distance between the Earth and the moon decreases when the Earth passes through the aphelion of the solar orbit.

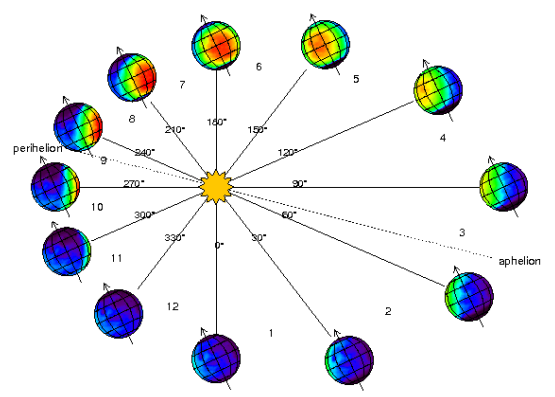


Due to the existence of the lunar spin in the lunar revolution, we know that the mass of orbital attraction of the lunar nucleus, always points the same face of the moon towards the Earth. This orbital rotation of the lunar revolution occurs due to the orbital attraction of the orbital masses of the Earth and the moon.

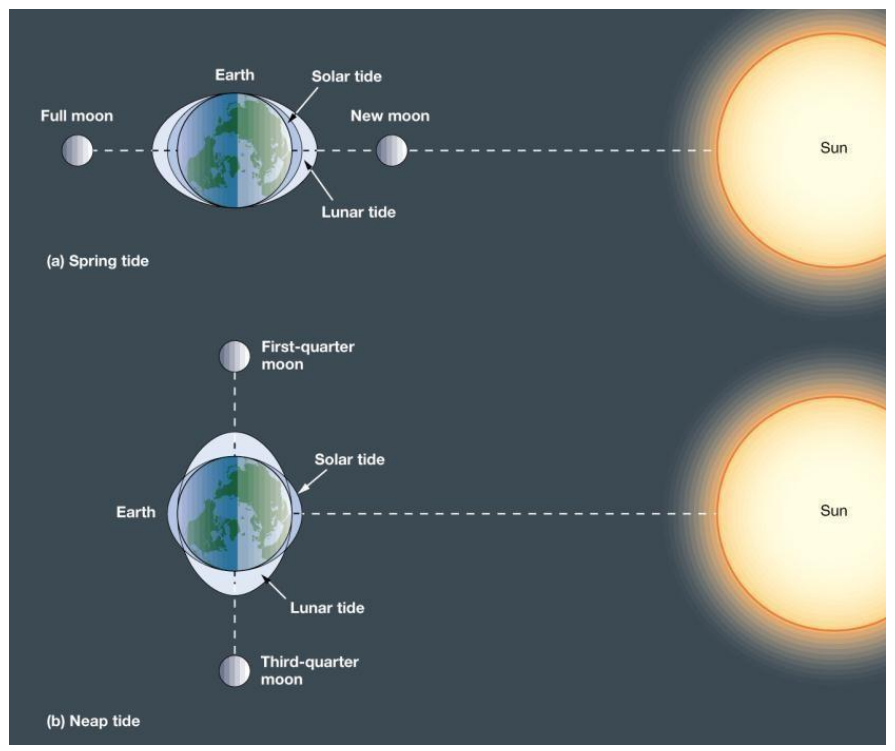

\section{Orbital superconductivity}

In 1602 Johannes Kepler reformed astronomy by geometric mathematics: because the planets sweep equal areas in equal times. With Kepler's second law of planetary motion, which reveals the findings of the model of elliptical orbits. However, Johannes Kepler could not explain the origin of the orbit, nor can he define the energy produced by the circular motion, the parabolic motion, the hyperbolic motion, and the elliptical motion of the orbit. Johannes Kepler, in the case of Mercury, then considered that the elliptical orbit has an acceleration in its orbital velocity, as the celestial body approaches the focus of the perihelion. But, in fact, Johannes Kepler was not able to understand that the orbital acceleration of Mercury in perihelion is due to solar heating of the nuclear mass of the nucleus of Mercury. Much later than Johannes Kepler, Eduardo Guimarães explained that the orbital superconductivity is generated by the nuclear energy of the geological nuclear mass of thermo-orbital repulsion with the geological nuclear mass of thermo-orbital attraction, that produces an elliptic orbital superconductivity.

In 2018, Eduardo Guimarães also explained that each star, planet, and moon has a nucleus containing three superconducting thermo-nuclear masses. Then the mixture of these three masses: geological nuclear mass of gravity attraction; geological nuclear mass of orbital attraction; geological nuclear mass of orbital repulsion, form the nuclear superconductor of the star, planet and moon nuclei.

The orbital superconductivity is a phenomenon that creates spatial orbital mathematics generated through the great orbital field of the Sun in the quantum and energetic transformation of the superconductive nuclear energy of the Sun's nucleus with the nuclei of the planets and their celestial bodies; and also of the superconductive nuclear energy of the planet's nucleus with the nucleus of the moon; and also of superconductive nuclear energy from the moon's nucleus with the Sun.

The mechanics of the solar system are given by the mass of superconducting nuclear energy. The mass of superconducting nuclear energy is concentrated in the nucleus of the sun, in the nucleus of the moon and in the nucleus of the celestial bodies and planets. 


\section{The orbital field of the solar system}

The great orbital energy field of the Sun has the shape of an orbital disk, but it is not visible.

The orbital field of the celestial bodies

In Saturn, Uranus and Neptune, we can see the orbital field. The orbital field is visible on Saturn, because of the large number of fragments of the orbital nuclear mass. The orbital field of the celestial bodies has the shape of an orbital disk.

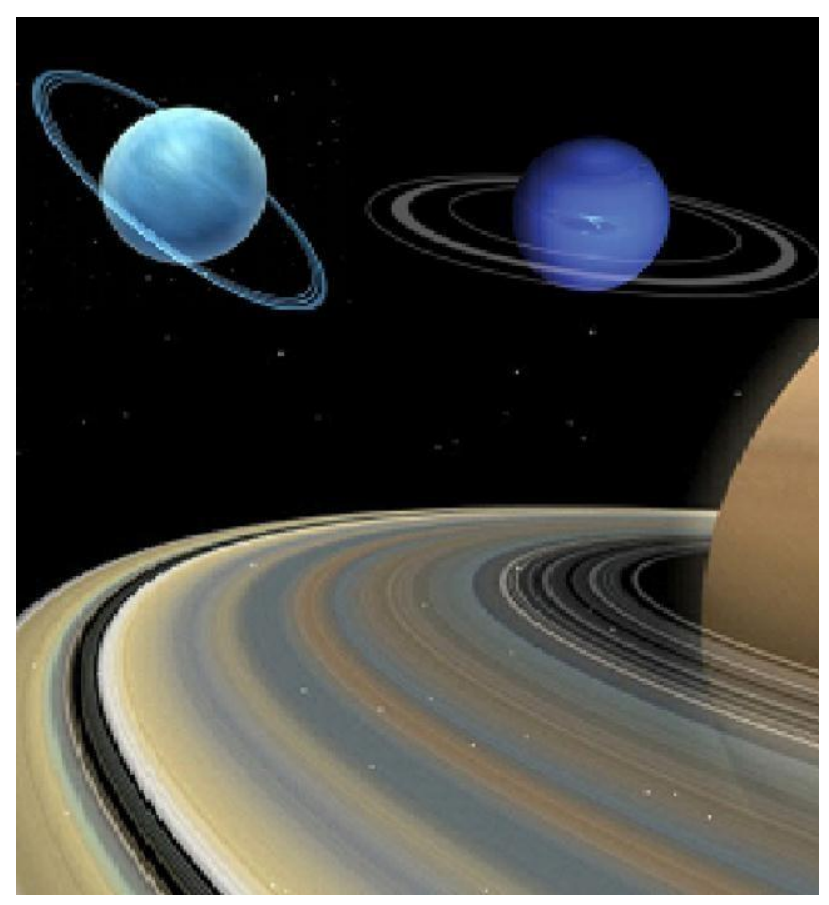

\section{The fragments of the orbital mass mixture}

The fragments of the orbital mass mixture form the rings of Saturn and generate the illustrative image of the disk of the orbital field.

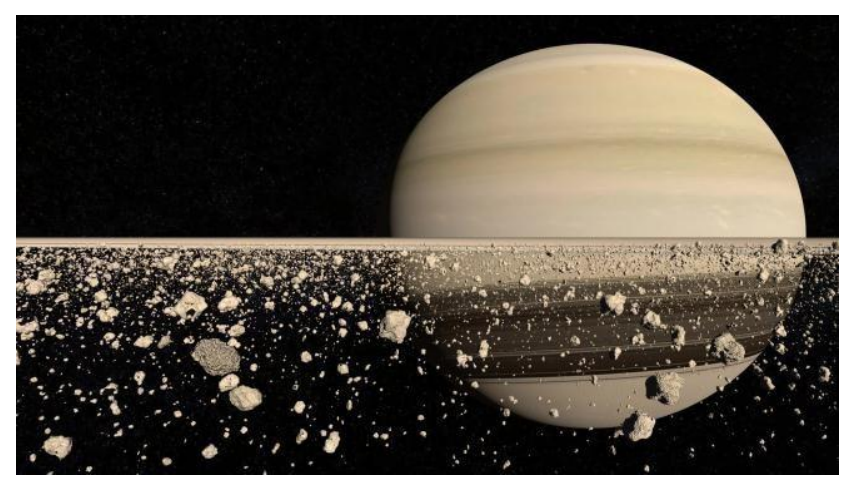

The micro-orbit of Mercury and the micro-orbit of the Moon

Because of the size of the small masses of the celestial bodies, Mercury and Moon. Then, Mercury and Moon create a micro-orbit around the celestial body of greater mass. 


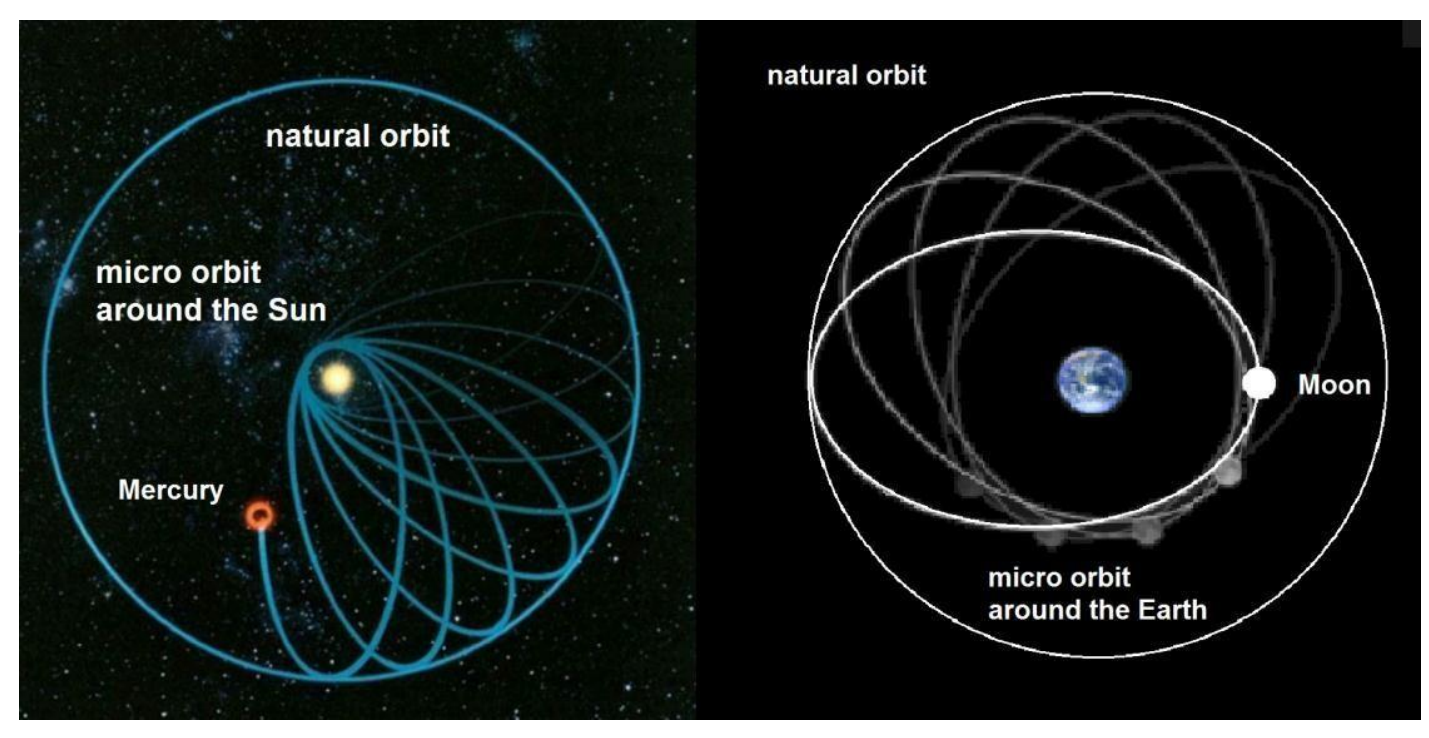

\section{Absence of orbit in the region of the moon surface and in the lunar space}

On the surface of the moon there is the field of spatial gravity, so that the orbital field can not coexist with the field of spatial gravity, because gravity attracts the celestial body to a cosmic collision. On Jupiter, Jupiter's fifth moon, when it entered Jupiter's atmospheric gravity field, fell and formed a huge abyssal crater on Jupiter.

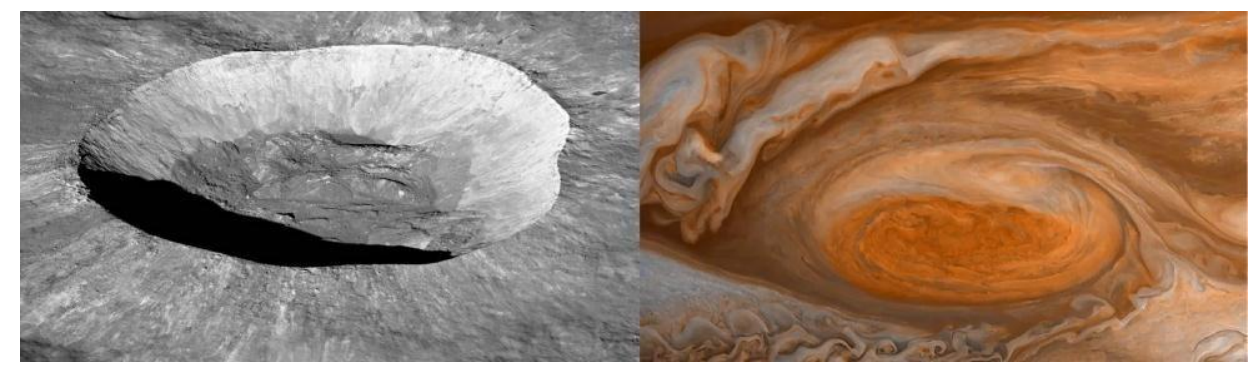

\section{The geometric curve of the orbit}

The orbital curves are the circle, ellipse, and parabola.

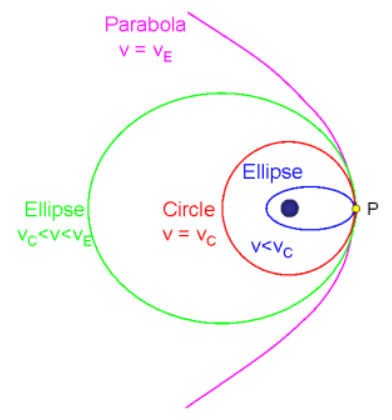

\section{Conclusion}

We conclude because of the existence of Mercury's micro-orbit, that Mercury will collide upon entering the field of the Sun's spatial gravity. So the solar system is self-destructive by the thermo-nuclear explosion produced by the cosmic collision of Mercury with the Sun and with the nucleus of the Sun. 
We conclude because of the existence of the Moon's micro-orbit, that the Moon will collide upon entering the field of Earth's atmospheric gravity. So the Earth is self-destructive by the thermo-nuclear explosion produced by the orbiting collision of the moon with the planet Earth.

We conclude that the three masses: geological nuclear mass of the attraction of gravity; geological nuclear mass of orbital attraction; geological nuclear mass of orbital repulsion that produce all the space-orbital mechanics of celestial bodies, planets, moons, artificial moons, stars, asteroids, comets, and, meteors.

We conclude that the three masses: geological nuclear mass of the attraction of gravity; geological nuclear mass of orbital attraction; the geological nuclear mass of the orbital repulsion that produce all the spaceorbital mechanics of the solar system.

We conclude that the three masses: geological nuclear mass of the attraction of gravity; geological nuclear mass of orbital attraction; the geological nuclear mass of the orbital repulsion that produce all the spaceorbital mechanics of the Milky Way.

We conclude that the three masses: geological nuclear mass of the attraction of gravity; geological nuclear mass of orbital attraction; geological nuclear mass of orbital repulsion create the three fields: Gravity Field, Field of Magnetism and Orbital Field.

We conclude that the nuclei of the moons, nuclei of the planets, nuclei of the stars, nucleus of the Sun, and the center of the Milky Way are formed by a thermonuclear superconductor capable of generating the natural energy fields in the form of: (gravity, orbit, magnetism).

We conclude that the sequential tremors of the big bang, produced deformations in the mass and formed giant liquid bubbles within the nucleus of the Milky Way; stars; planets.

We conclude that these giant liquid bubbles within the nucleus of the Milky Way produced many billions of stars with different sizes and different energy levels of the star nucleus.

We conclude that these giant liquid bubbles inside the nucleus of the stars produced sequences of planets with different sizes and different level of energy of the planet's nucleus.

We conclude that these liquid bubbles within the nuclei of the planets produced sequences of moons of different sizes and different levels of energy from the moon's nucleus.

We conclude that the moons are celestial bodies of geological origin of the sequential fourth generation of the big bang. Therefore, the moon has a small mass and its nucleus has low amount of energy generating: orbit, gravity, magnetism and heat.

We conclude that the moon lost the kinetic energy, the axial rotation movement and stopped at uncertain date.

We conclude that the gravity originates from the nuclear energy generated by the geological nuclear mass of the attraction of gravity.

We conclude that the gravitational energy of the moon generates the field of lunar gravity that is divided into zones with microgravity; spatial gravity; surface gravity; underground gravity; and nucleus gravity.

We conclude that the parabola is the gravitational curve that is visible through the mass curve.

We conclude that in the great dimension of the space there is no gravity, however, there is gravity in the space field and in the atmospheric field of the celestial bodies that contain geological mass of attraction of gravity.

We conclude that on the moon, energy is naturally transformed into a magnetic field. 
We conclude that the nuclear magnetic field of the moon is weak and is not able to bend the light.

We conclude that the geometric curve of magnetism is an invisible nephroid curve extending between the two north and south poles.

We conclude that in the core of the moon, energy is naturally transformed into an orbital energy field.

We conclude that the equilibrium of the orbital superconductivity pathway is generated in the total mass of the moon by the energy of the geological nuclear mass of thermo-orbital repulsion that subtracts the energy from the geological nuclear mass of thermo-orbital attraction and thus produces an elliptical geometry curve of orbital superconductivity.

We conclude that the orbital superconductivity is a phenomenon that creates spatial orbital mathematics generated through the great orbital field of the Sun in the quantum and energetic transformation of the superconductive nuclear energy of the Sun's nucleus with the nuclei of the planets and their celestial bodies; and also of the superconductive nuclear energy of the planet's nucleus with the nucleus of the moon; and also of superconductive nuclear energy from the moon's nucleus with the Sun.

We conclude that the great orbital energy field of the Sun has the shape of an orbital disk, but it is not visible. We conclude that the orbital curves are the circle, ellipse, parabola and hyperbola.

\section{References}

$1 \quad$ Edwin Arthur Burtt.; (2003). "The Metaphysical Fondations of Modern Science". Dover Publications Inc.

2 Eduardo Santos Guimarães.; (2018). "Theory of Nuclear Universe and the Orbital Supercondutivity of Eduardo Guimarães". Independently published.

3 Sir Isaac Newton.; (1687). "Philosophiae Naturalis Principia Mathematica".

$4 \quad$ Alan Lightman.; (2009). "The Discoveries: Great Breakthroughs in 20th-Century Science, Including the Original Papers". Vintage.

$5 \quad$ Jerome G. Morse.; (2013). "Nuclear Methods in Mineral Exploration and Production". Elsevier.

6 Stephen Hawking.; (2007). "A Briefer History of Time". Bantam.

$7 \quad$ Eduardo Santos Guimarães.; (2018). "The nuclear universe of orbital superconductivity, the orbital movements of the Solar System and the secret of the supernova". Independently published.

$8 \quad$ Tidels.; (2018). "Origin of the Universe: The Big Bang Theory".

$9 \quad$ Hermann Minkowski.; (2016). "Space and Time: Minkowski's Papers on Relativity". Minkowski Institute.

10 Albert Einstein.; (2014). "Relativity: The Special and the General Theory". General Press.

11 Laurent-Patrick Levy.; (2013). "Magnetism and Superconductivity (Theoretical and Mathematical Physics)". Springer.

12 Gillian Turner.; (2011). "North Pole, South Pole: The Epic Quest to Solve the Great Mystery of Earth's Magnetism". The Experiment.

13 Codex Regius.; (2017). "Ceres: Pluto's little sister: The Dawn spacecraft over the volcanoes of a dwarf planet". Amazon Servicos de Varejo do Brasil Ltda. 
14 Charles D. Dermer.; (2009). "High Energy Radiation from Black Holes: Gamma Rays, Cosmic Rays, and Neutrinos (Princeton Series in Astrophysics)". Princeton University Press.

15 Walter D. Loveland.; (2017). "Modern Nuclear Chemistry". Wiley.

16 David Branch.; (2017). "Supernova Explosions". Springer.

17 Steven J Seferi.; (2018). "Astronomy Orbits of the Planets". Createspace Independent Publishing Platform.

18 Distinctive Journals.; (2018). "Science Journal Space Station Orbits Planet". Fairleigh Dickinson Univ Pr.

19 Eduardo S. Guimarães; (2018). "The beginning of the nuclear universe ans the theory of orbital superconductivity of the celestial bodies". Journal of Advances in Physics. ISSN2347-3487

20 Holly Duhig; (2018). "The sun". Booklife.

21 Linda T. Elkins-Tanton. (2010). "The Sun, Mercury, and Venus". Facts on File.

22 Dawn M Angrove. (2011). "Magnetite". Nova Science Publishers.

23 Einar Lindeman. (2018). "Magnetite Occurrences Near Calabogie, Renfrew County, Ontario". Forgotten Books.

24 George Leander Chapin. (2017). "Chapin's System of Planet Movement". Forgotten Books.

25 Jeffrey Kluger. (2018). "Apollo 8". Picador.

26 V.I. Ferronsky. (2015). "Nuclear Geophysics". Springer.

27 Kris Davidson. (2014). "Eta Carinae and the Supernova Impostors". Springer.

28 James B. Hartle. (2016). "Gravity: An Introduction to Einstein's General Relativity". Addison-Wesley Professional.

29 George Gamow. (2003). "Gravity". Dover Publications.

$30 \quad$ Science News. (2016). "Einstein's Gravity". Diversion Books.

31 Francisco Fernflores.; (2017). "Einstein's Mass-Energy Equation". Momentum Press.

32 Herbert Goldstein.; (2014). "Classical Mechanics". Pearson.

33 HardPress.; (2018). "Centrifugal Force and Gravitation". HardPress.

34 Anthony Burke.; (2017). "Uranium (Resources)". Polity.

35 Wolfgang Hoffelner.; (2012). "Materials for Nuclear Plants". Springer.

36 Walter Minto.; (2018). "Researches Into Some Parts of the Theory of the Planets, in Which is Solved the Problem, to Determine the Circular Orbit of a Planet". Gale ECCO, Print Editions.

37 Yang Yu.; (2016). "Orbital Dynamics in the Gravitational Field of Small Bodies". Springer.

38 Charles D. Ferguson.; (2011). "Nuclear Energy". Oxford University Press.

39 R.K. Verma.; (2012). "Gravity Field, Seismicity and Tectonics of the Indian Peninsula and the Himalayas". Springer.

$40 \quad$ Nicholas Tsoulfanidis.; (2012). "Nuclear Energy: Selected Entries from the Encyclopedia of 
Sustainability Science and Technology". Springer.

41 I.S. Roxburgh.; (2012). "Geology of High-Level Nuclear Waste Disposal". Springer.

42 W. Heisenberg.; (2015). "Nuclear Physics". Philosophical Library.

43 T.J. Mahoney.; (2014). "Mercury". Springer.

44 Georgia Beth.; (2018). "Discover Mercury". Lerner Classroom.

45 Kevin H. Baines.; (2018). "Saturn in the 21st Century". Cambridge University Press.

$46 \quad$ W. G. Sebald.; (2016). "The Rings of Saturn". W. W. Norton \& Company.

47 Science News.; (2016). "Dimensions of Time". Diversion Books.

48 Brian Greene.; (2009). "The Elegant Universe". W. W. Norton \& Company.

49 Johannes Andersen.; (2009). "The Galaxy Disk in Cosmological Context". Cambridge University Press.

50 R. S. de Jong.; (2007). "Island Universes". Springer.

$51 \quad H o n g-X i n$ Zhang.; (2018). "Stellar Disk Evolution and Gaseous Disk Turbulence of Dwarf Irregular Galaxies". Springer.

52 Carl Sagan.; (2013). "Cosmos". Ballantine Books.

53 Margaret J Goldstein.; (2018). "Discover Jupiter". Lerner Publications.

54 Stuart Clark.; (2018). "The Unknown Universe". W. W. Norton \& Company.

55 Gene Kranz.; (2009). "Failure Is Not an Option: Mission Control from Mercury to Apollo 13 and Beyond". Simon \& Schuster.

56 Héctor González Sarrià.; (2016). "PHOBOS". Amazon Servicos de Varejo do Brasil Ltda.

57 Eduardo Santos Guimarães.; (2018). "The theory of the nuclear universe and the secret of the supernova". JOURNAL OF ADVANCES IN PHYSICS.

58 Eduardo Santos Guimarães.; (2018). "THE FALL THE OF MOON OF JUPITER AND THE FORMATION OF THE RINGS OF SATURN". Independently published.

59 William Brehm.; (2014). "William Gilbert". Amazon Servicos de Varejo do Brasil Ltda.

60 NASA.; (1997). "Microgravity: NASA Educator Guide". Amazon Servicos de Varejo do Brasil Ltda.

61 Buzz Aldrin.; (2015). "Return to Earth". Open Road Media.

62 Thomas Posch.; (2017). "Johannes Kepler: Die Entdeckung der Weltharmonie". wbg Theiss.

63 M. Date.; (2012). "High Field Magnetism". North Holland.

64 William H. Waller.; (2017). "The Milky Way - An Insider`s Guide". Princeton University Press.

65 Christopher Riley.; (2010). "Apollo 111969 Owners' Workshop Manual". Haynes Publishing.

66 David M. Harland.; (2009). "The First Men on the Moon: The Story of Apollo 11". Springer.

67 National Aeronautics and Space Administration (NASA).; (2015). "Shooting the Moon: How NASA

Found Water on the Moon, the LCROSS Mission - Lunar Crater Observation". Progressive Management. 
Bradley M. Peterson.; (1997). "An Introduction to Active Galactic Nuclei". Cambridge University

Press.

69 Brian Punsly.; (2008). "Black Hole Gravitohydromagnetics". Springer.

70 Gerard F. Gilmore.; (2013). "Planets, Stars and Stellar Systems". Springer. 


\section{Annex 1 - The nature of the time of the moon}
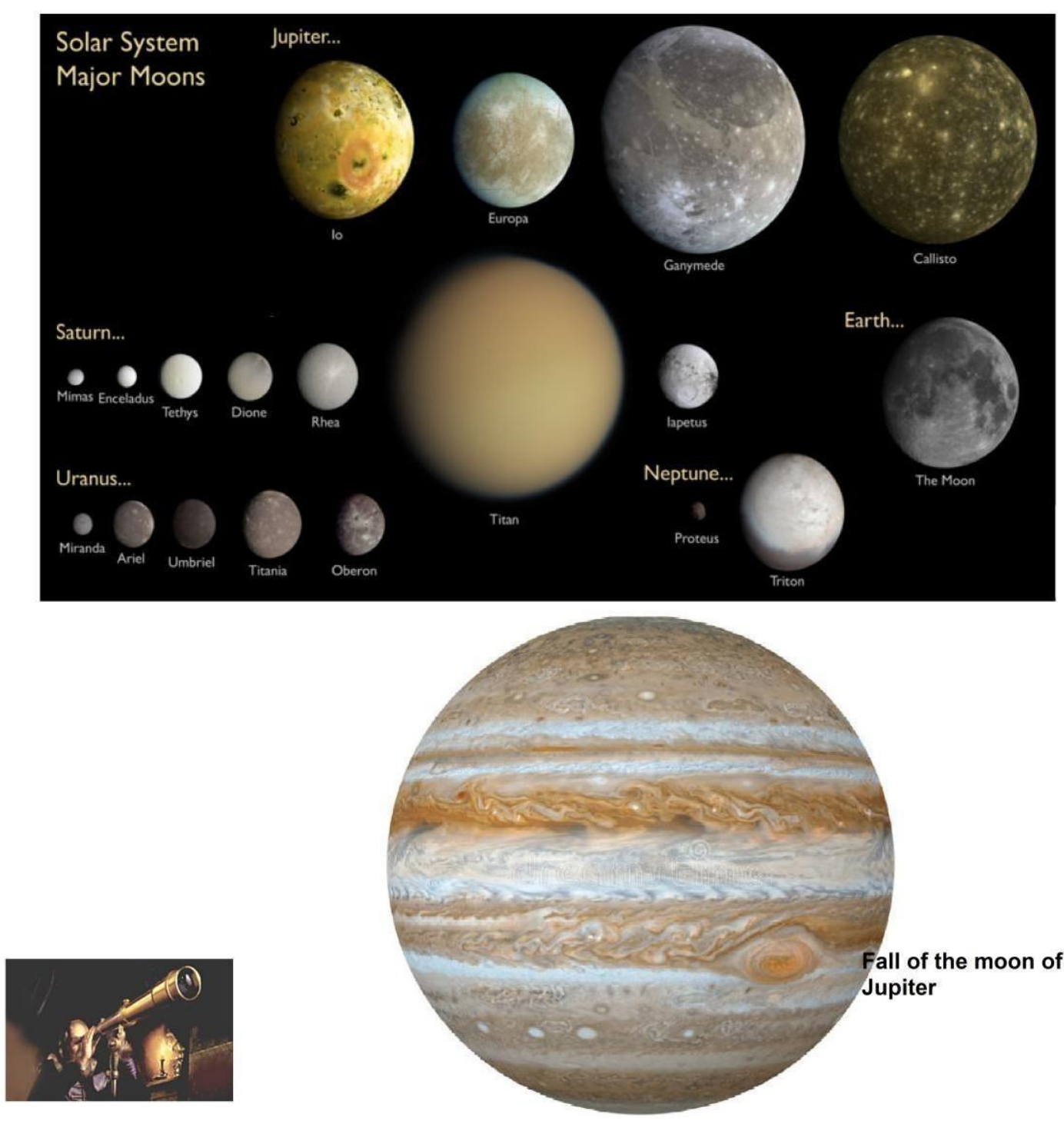

\section{How did the moon arise?}

After the Big Bang of the primitive universe. Thermonuclear heat produced a nuclear self-ignition of the galaxy's primitive thermonuclear mass, the Milky Way. Then there were numerous hyper tremors and numerous deformations of the mass of the galaxy's nucleus.

These deformations of the nucleus of the galaxy have become super-bubbles of nuclear mass of the galaxy nucleus and by the action of super-fissure in the mass of the nucleus of the galaxy.

Then, these deformations in the form of mass super-bubbles of the nucleus were repelled in space through the geological nuclear mass of orbital repulsion, and thus, these super bubbles of liquid mass were equal in number to the celestial stars of the Milky Way.

Then there were new deformations and fissures of the mass of the nucleus of all stars in the galaxy and also in the Sun, and then the mass of orbital repulsion expelled super bubbles and large bubbles of liquid mass equal to the number of planets in the solar system.

Then there were new deformations and fissures of the mass of the planet's nucleus, and then the mass of orbital repulsion expelled small bubbles of liquid mass equal to the number of moons of the orbital system of each planet. 


\section{The Big Bang}

The big bang was the immense thermonuclear explosion of the early universe that contained the three nuclear masses of the cosmos: the nuclear mass of attraction of gravity; the nuclear mass of orbital attraction; the nuclear mass of orbital repulsion;

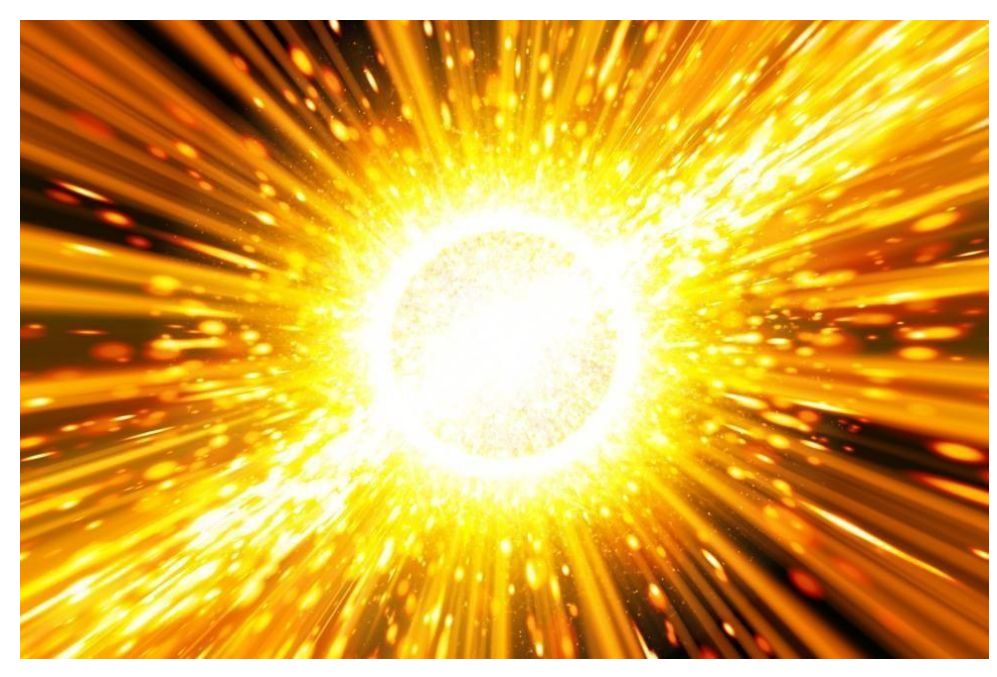

\section{The time of the Big Bang}

The time of the big bang is the count of the new chronological time, because of the natural creation of the nuclear universe of the sidereal space by the total destruction of the primitive nuclear universe.

\section{The vacuum and space of the cosmos}

The big bang consumed all the matter of the primitive universe in the gaseous state and much of the nuclear matter in the liquid state, producing the vacuum and the spatial dimension of the cosmos.

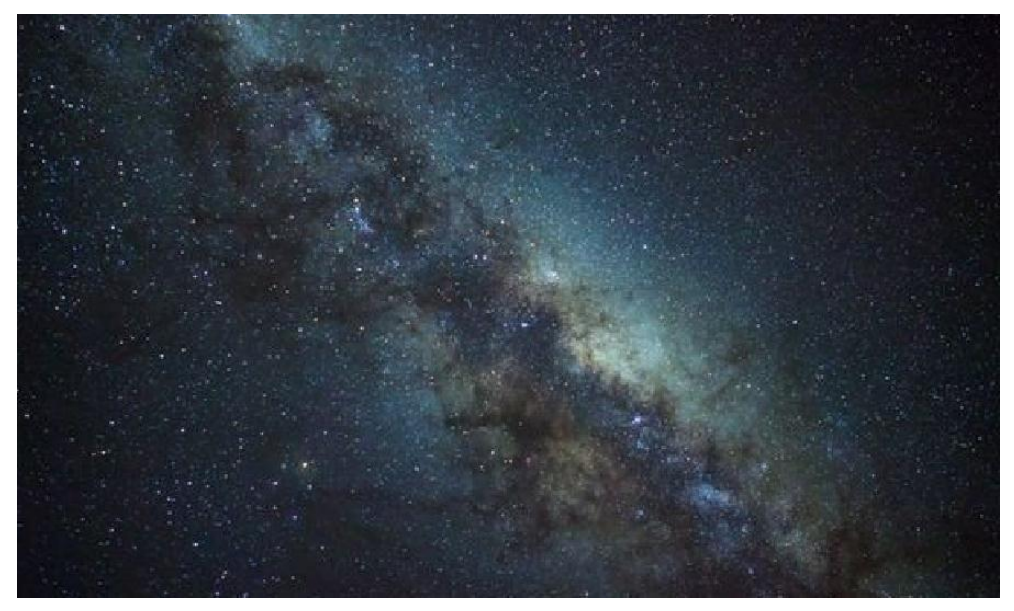

The auto ignition of the nuclear universe in the sidereal space.

The heat of the big bang produced the auto-ignition of the galactic nuclear masses. Then the galactic auto ignition began the process of galactic disk formation. 


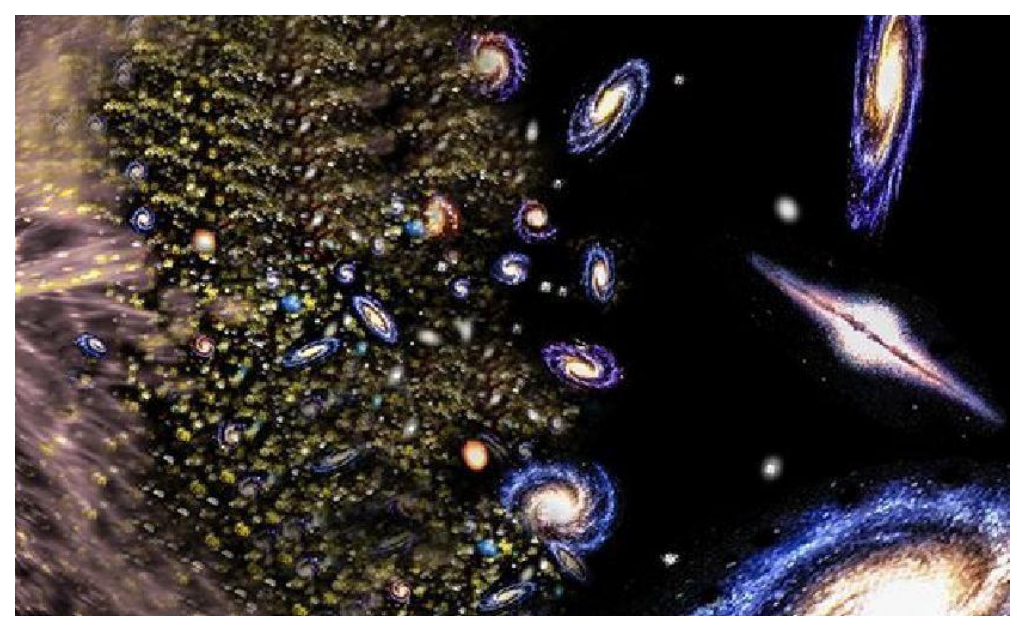

The creation of bubbles in the great shake of space

Because of the space shaking the mass of the nucleus of the celestial bodies in the liquid state, then the space tremor creates internal cracks, which become mass bubbles of the nucleus.

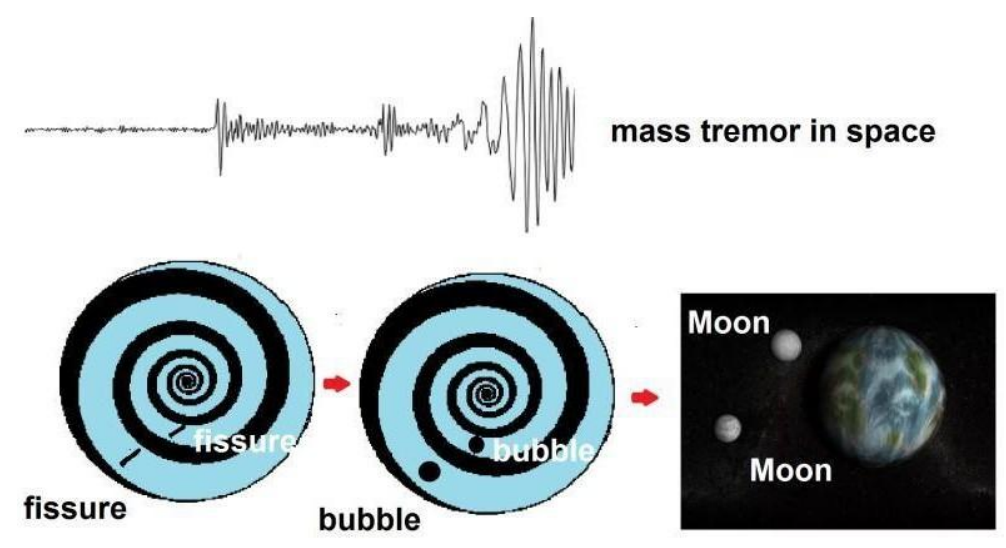

\section{Deformation of the mass of the nucleus of the Milky Way;}

Due to the liquid state property of bubble generation in space, there was a natural deformation of the galactic nucleus that generated the fissure of the mass of the galactic nucleus, and resulted in the immense amount of stars of the galactic disk.

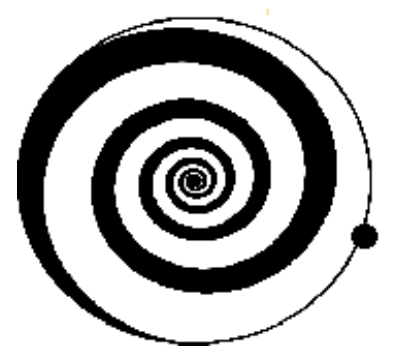

\section{Deformation of stellar mass in the galaxy's nucleus}

The deformation of the galactic disk architecture

The deformation in the architecture of the galactic disk occurs due to the creation of many billions of super 
bubbles that give the dimension of the galactic orbital field.

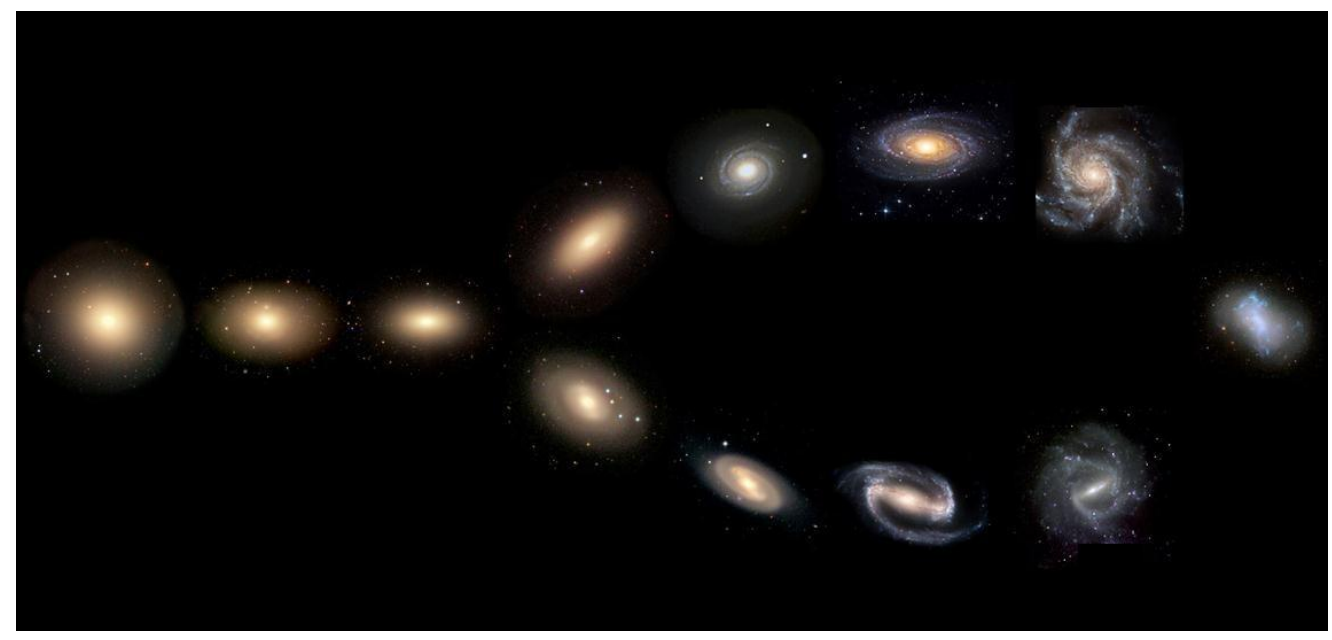

\section{The life in the galaxy, Milky Way}

The closer the celestial star is to the galactic center, or "galactic nucleus," the greater the trepidations and cosmic nuclear shudder suffered in the time of the Big Bang. The nuclear trepidations of the big bang made billions of fissures in the nucleus of the galaxy, which became billions of super bubbles of net mass of stars. Through the nuclear mass of orbital repulsion the billions of stellar mass super bubbles created the geometric architecture of the galactic disk of the Milky Way.

The bigger the trepidations of the big bang, the bigger the super bubble of the celestial star. (star, planet, moon).

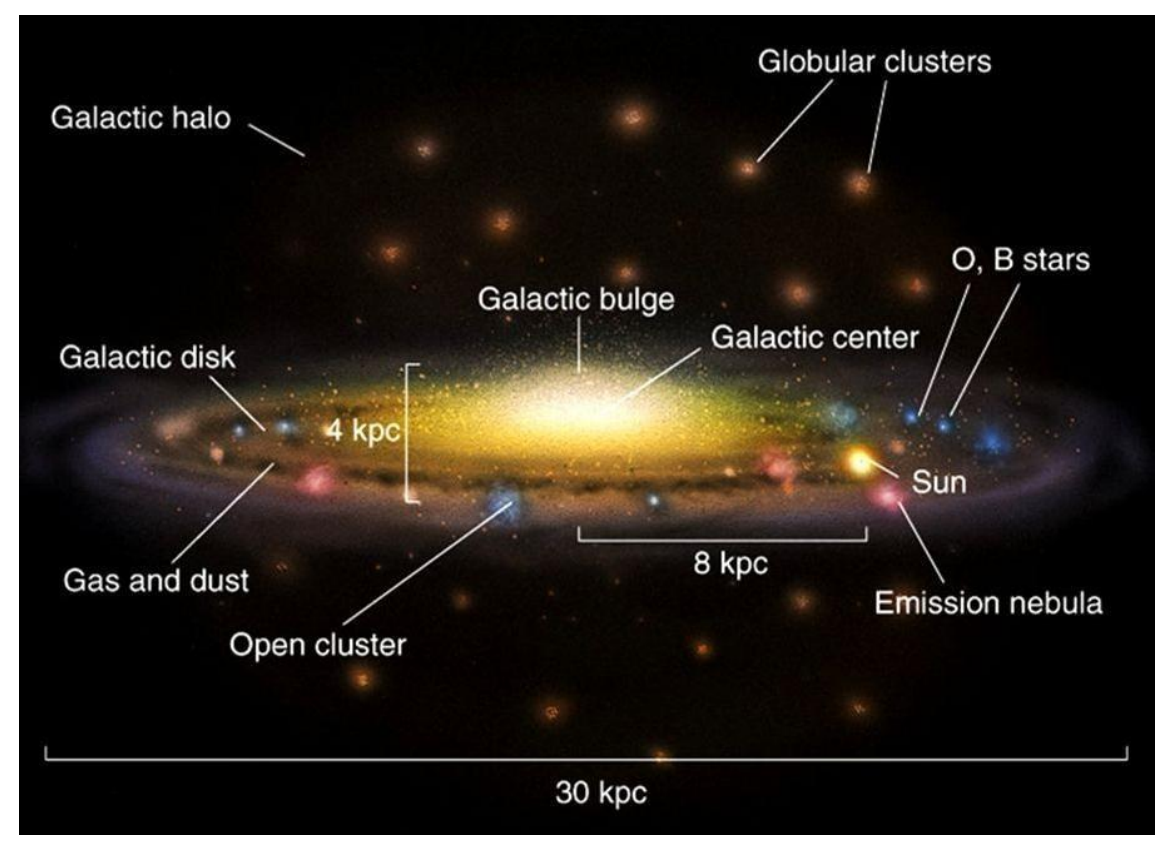

\section{The geometric dimensions of the Orion arm nuclear tremors}

At the time of the Big Bang, Orion's arm had at least three major nuclear explosions that generate the super-giant stars: Gama Cruxis, then another great explosion generated Rigel and Canopus, and another 
great explosion spawned Aldebahan.

Two much smaller nuclear explosions generated the great stars Alnitak and Arcuturus and another much less nuclear explosion generated the star Spica, and another much smaller nuclear explosion generated the stars Vega, Sirius and Sun among many other stellar mass super bubbles.

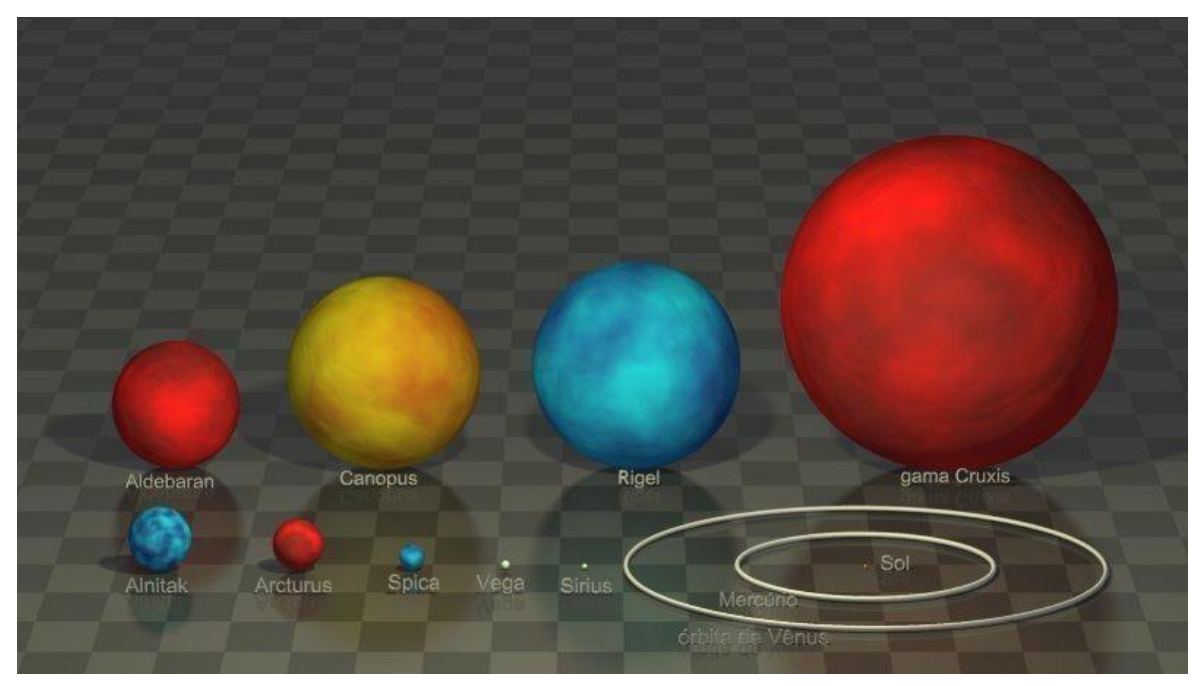

\section{The geometric dimensions of the nuclear tremors of the Solar System}

After the Big Bang, and the birth of the Sun, the solar system had at least two major nuclear explosions that spawned the two pairs of super planets (Neptune and Uranus), and (Saturn and Jupiter). Another two smaller nuclear bursts generated the two pairs of small planets (Ceres and Mars), and (Earth and Venus), and several moons

A fifth nuclear explosion of the big bang, much smaller, may have spawned the planet's moon (Earth and Mercury). So, Mercury is a moon of Venus.

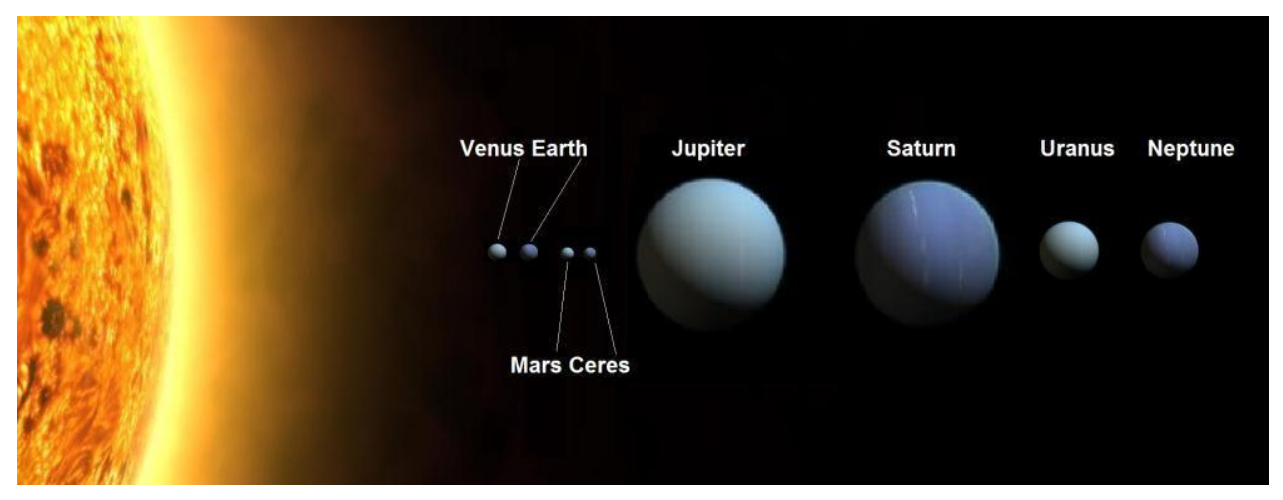

\section{The planet Ceres was destroyed after the fall of Jupiter's moon}

In the space in the place of Ceres we find vestiges of Ceres and a belt of asteroids. 


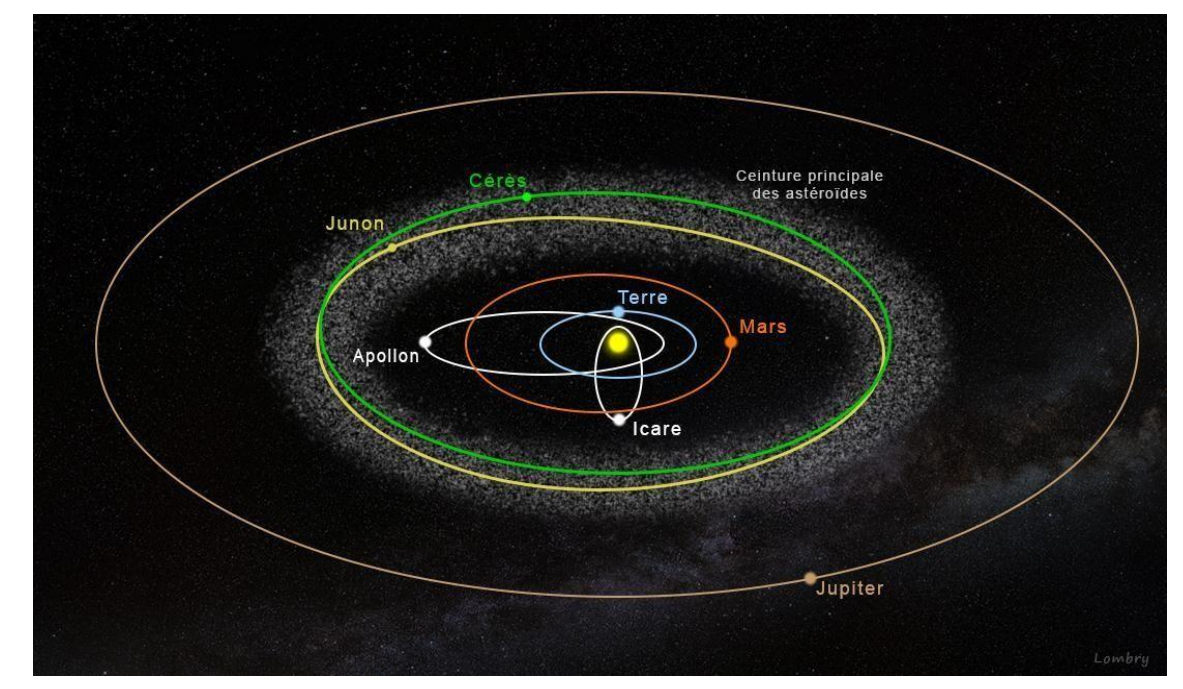

\section{The comparison between the dwarf planet and the giant planet}

The moon is a small celestial body with geometric dimensions of a dwarf planet.
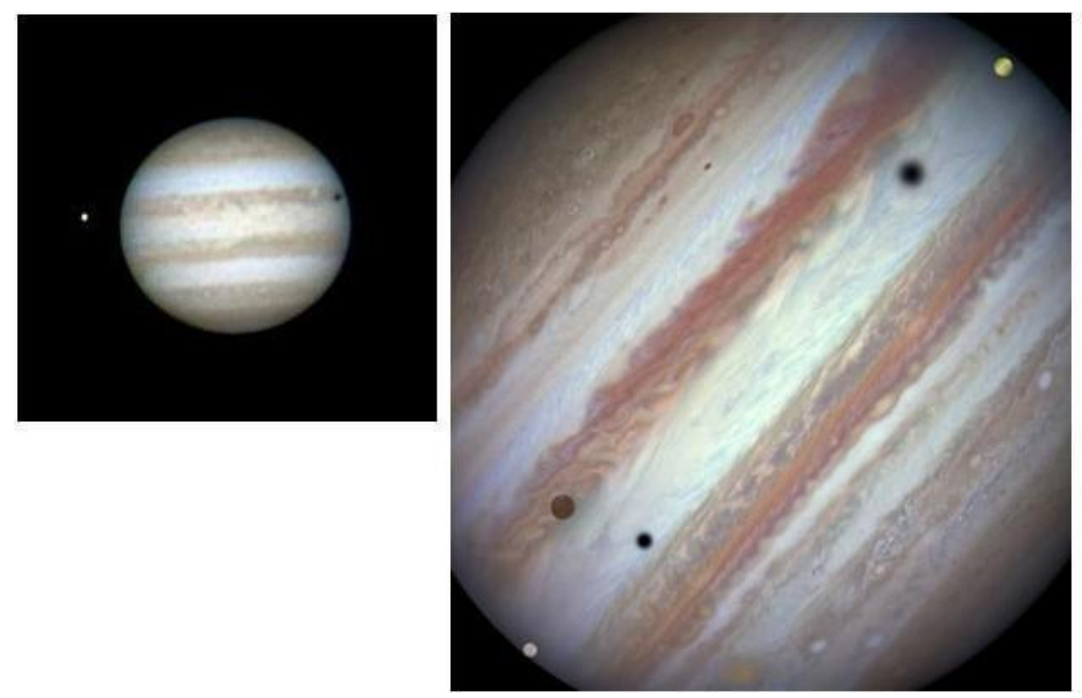

The thermonuclear energy of the moon's nucleus gravity falls from energy level due to the solidification of the nuclear mass of the lunar nucleus.

The time passes at the speed of light. Then the thermonuclear energy of the moon's nucleus gravity dissipates and becomes insufficient to produce the kinetic energy of the lunar spin motion due to the solidification of the mass of the nucleus. Then, the moon loses speed and stops the 360-degree rotation movement. 


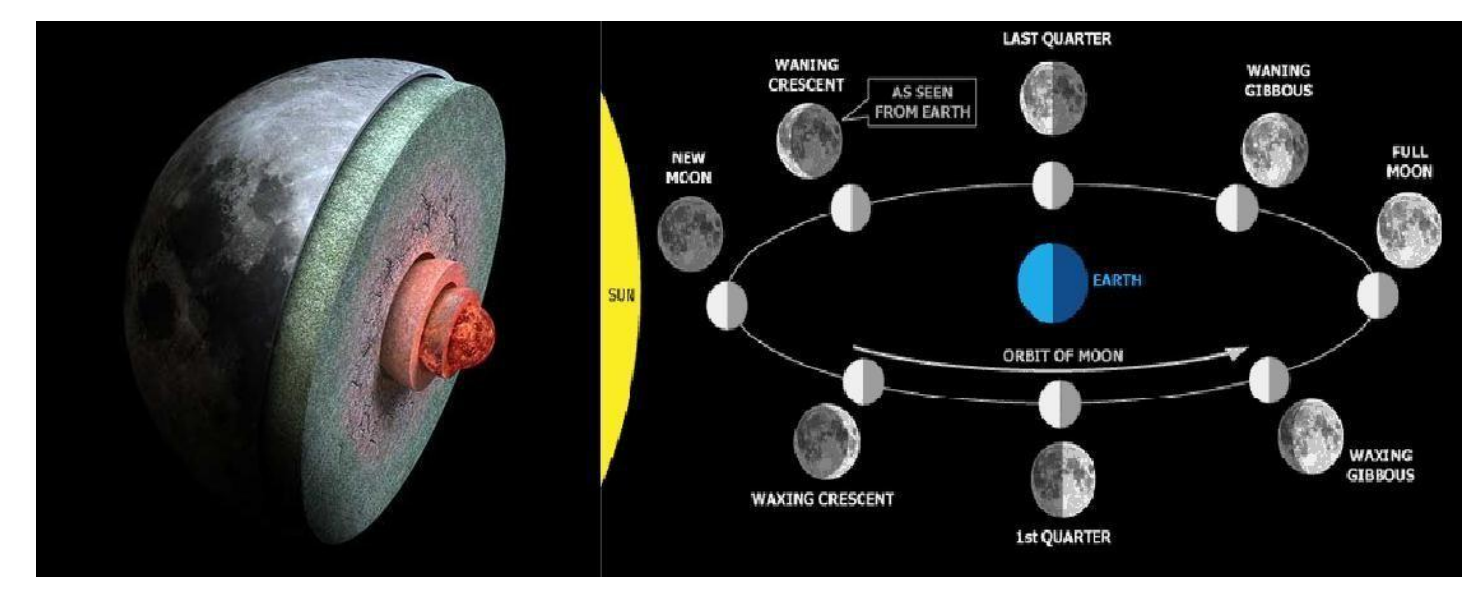

The thermonuclear energy of the orbital repulsion of the moon nucleus falls level due to the dissipation of the thermonuclear energy of the nucleus

Time continues to pass at the speed of light. Then the thermonuclear energy of the orbital repulsion of the moon's core dissipates and again becomes insufficient to produce the lunar distancing orbital repulsion energy, because of the reduction of the energy of the nucleus mass. Then, the moon creates an orbital approximation curve named of super moon, for the future collision with the planet.

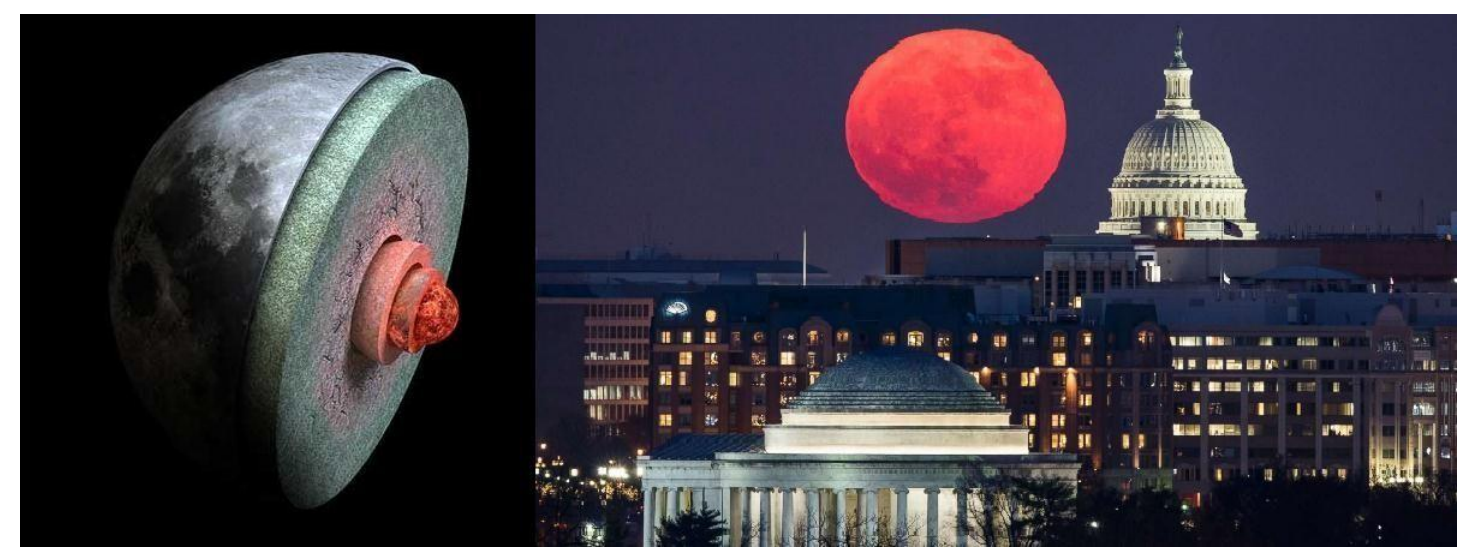

The super dimension of the moon is caused by the chronological period of approach of the super moon

When the moon does the revolution movement during the elliptic curve distancing at the aphelion, that is, each chronological period of approximately 414 days or approximately 14 months. The mass of orbital attraction of the lunar nucleus is attracted by the equation of the masses of orbital attraction of the Earth's nucleus, aligned with the nucleus of the Sun. Then we can verify the super moon, through the loss of energy of the mass of orbital repulsion of the nucleus of the moon. 


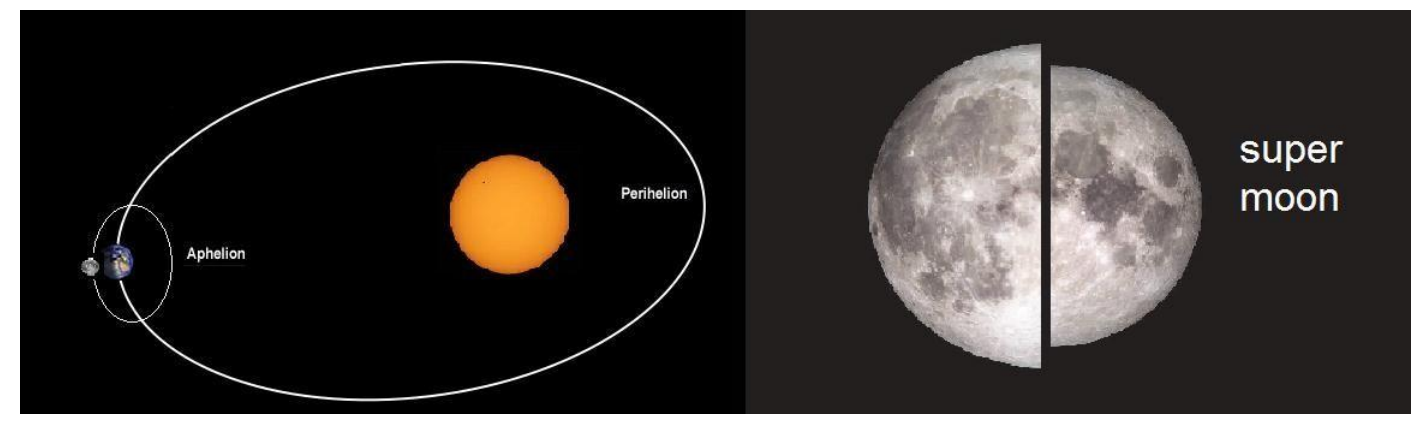

Table of the beginning of the fall of the moon

\begin{tabular}{|c|c|c|c|c|c|}
\hline $\begin{array}{l}\text { Number of super moons } \\
\text { per period }\end{array}$ & First super moon (full) & Second super moon (full) & Third super moon (full) & Start period & End period \\
\hline 01 & $03 / 19 / 2011$ & & & & \\
\hline 01 & $05 / 05 / 2012$ & & & 413 days & \\
\hline 01 & $06 / 23 / 2013$ & & & 414 days & \\
\hline 03 & $07 / 13 / 2014$ & $08 / 10 / 2014$ & $09 / 09 / 2014$ & 385 days & 443 days \\
\hline 03 & $08 / 29 / 2015$ & $09 / 28 / 2015$ & $10 / 27 / 2015$ & 412 days & 471 days \\
\hline 03 & $10 / 16 / 2016$ & $11 / 14 / 2016$ & $12 / 14 / 2016$ & 414 days & 473 days \\
\hline 03 & $12 / 03 / 2017$ & $01 / 02 / 2018$ & $01 / 31 / 2018$ & 413 days & 472 days \\
\hline
\end{tabular}

1) In the years prior to 2011, we find RARE APPARITIONS OF THE SUPER MOON;

2) In the years 2011 to 2013, we find CONSTANT APPARITIONS OF (1) SUPER MOON FOR A PERIOD OF 414 DAYS;

3) In the years 2014 to 2017, we find CONSTANT APPARITIONS OF (3) SUPER MOON FOR A PERIOD OF 414 DAYS.

4) Because the sequence of (3) months has invaded the year of 365 days. Then, in 2018 the period of 414 days, was compromised by the invasion of 2017 on $01 / 02 / 2018$ and 01/31/2018.

\section{Graph of the beginning of the fall of the moon}

From the date table we put the chart below that shows the accelerated fall of the moon in the approximation period in 412 days. This chart shows that by 2010 there was no super moon, and that super moon phenomenon appeared in 2011 , and fixed itself on the astronomical calendar in a unique apparition that ranges from 412 days to 414 days. 
To our surprise, we realized that in 2014 a new phenomenon occurred to close the orbital curve of the moon that produced three super moons.

Strangely, this three super-moon phenomenon that makes a period between 385 days and 414 days, has been fixed itself again in the astronomical calendar. So we predicted that by 2030 there will be a super super moon, much bigger than the super moon of 2011, which demonstrates the accelerated descent of the lunar orbital curve, as shown below:

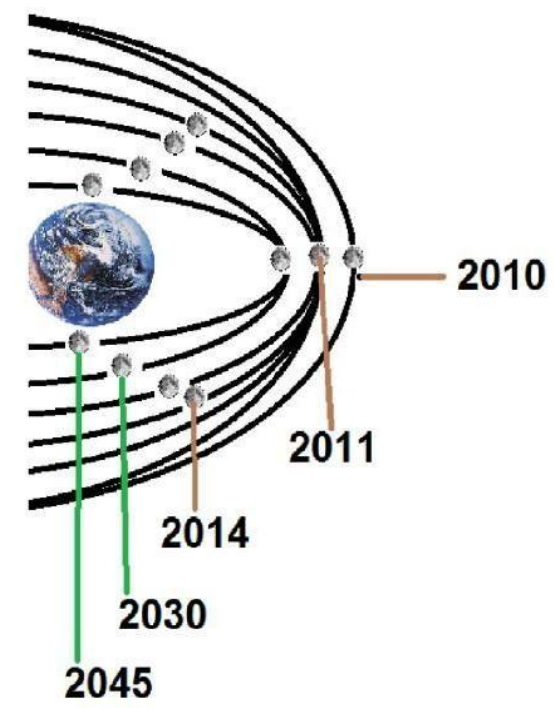

\section{Conclusion}

We conclude that before the Big Bang, the primitive universe brought together a high level of mass of nuclear energy, but without the ignition of the heat of the stars, and also without species or type of life.

We concluded that the Big Bang, created a new count for the time.

We conclude that the Big Bang created hyper tremors that fissured the nuclear mass inside galaxies. We conclude that super tremors fissured the nucleus of stars, and planets.

We conclude that the Milky Way suffered the tremors of the Big Bang explosions.

We conclude that the mass of the nucleus of galaxies has generated many billions of stars within the galactic disk.

We conclude that the mass of the nucleus of the galaxies expel numerous billions of stars that formed the galactic disk.

We conclude that the mass of the nucleus of galaxies, stars and planets suffered deformations by the Big Bang, and these deformations created the dimension of the stars, planets, and the dimensions of the moons.

We conclude that the mass of a hyper-bubble generated the Milky Way.

We conclude that numerous hyper-tremors have hurt and fissured the nuclear mass of galaxies.

We conclude that numerous super bubbles of liquid mass have become equal in number to the celestial stars of the Milky Way. 
We conclude that the Sun originated from a super-bubble of the nucleus of the Milky Way.

We conclude that the Earth and the other planets originated from the super bubbles and large bubbles of the Sun's nucleus.

We conclude that each moon originated from small bubbles of liquid mass of the nucleus of the nearest planet.

We conclude that the big bang was the immense thermonuclear explosion that extinguished the primitive universe.

We conclude that the vacuum of cosmic space was produced by the sequence of the huge explosion of the big bang.

We conclude that the big bang created the spatial macro-dimensions of the cosmos. We conclude that the big bang created the self-ignition of all galaxies in the universe. We conclude that the big bang created the sidereal space.

We conclude that the big bang created the hyper shake and tremor of space.

We conclude the existence of a new physical property of the space capable of deformation or generation in the form of bubble of mass, when the mass is in the gaseous state and in the liquid state.

We conclude that the sequence of Big Bang nuclear super explosions deformed the architecture of the galactic disk, and generated all the geometric dimensions of matter for all and any matter in the galaxy.

We conclude that the greater the trepidations of the big bang, the bigger the super bubble.

We conclude that the moon arose because of a distant nuclear explosion, or a small nuclear explosion near the solar system.

We conclude that the moon arose through a great fissure of the nucleus of the planet.

We conclude that the moon is a celestial body of the fourth generation of nuclear explosions of the Big Bang.

We conclude that the Sun is a celestial body, of the second generation of nuclear explosions of the Big Bang.

We conclude that the moon, having arisen in the fourth generation, is a heavenly body poor in minerals, and with little energy.

We conclude that the moon is a celestial body with a low field of gravity. We conclude that the moon is a celestial body with a low magnetic field. We conclude that the moon is a celestial body with a low orbital field.

We conclude that the moon has low heat in the nucleus.

We conclude that the nucleus of the moon is in the process of reducing temperature and solidifying the nuclear mass.

We conclude that the moon stopped by the loss of kinetic energy.

We conclude that the heat of the nucleus of the moon is in the irreversible process of thermonuclear dissipation.

We conclude that the super moon is the spatial point of deformation and approximation of the orbital curve that generates the spatial distance between the moon and the Earth. 
We conclude that the super moon is the spatial point of deformation and future fall of the orbital curve of the moon.

We conclude that the super moon is the space point of future orbital collision, except for some process of anticipation of the collision clock.

We conclude that the super moon is a phenomenon of nuclear energy loss of orbital repulsion and loss of orbital distance.

We conclude that the super moon is a phenomenon of physics with orbital astronomical effect.

We conclude that the super moon is a phenomenon of test of the loss of the energy of orbital repulsion of the moon, that begins in the period between 412 days to 414 days and ends in the period between 471 days to 473 days.

\section{Annex 2 - The loss of energy, the impact craters, the desertification of moon water sources, and the total desertification of the seas of the moon.}

\section{The water of the moon}

On the moon the water is deposited in underground basins. As the moon's subterranean gravity is not much greater than

$1.62 \mathrm{~m} / \mathrm{s}^{2}$, therefore, on the moon there are no natural water sources and natural waterfalls.
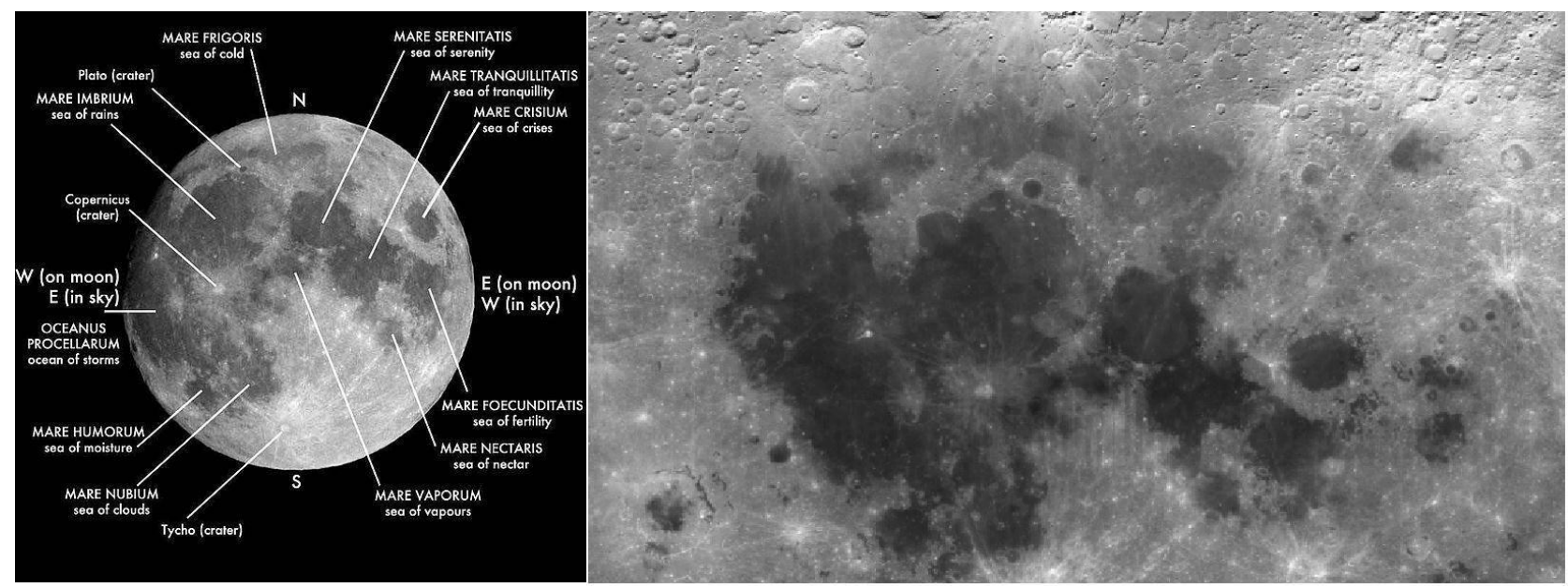

\section{The basins of groundwater of the moon}

The water of the moon is underground.

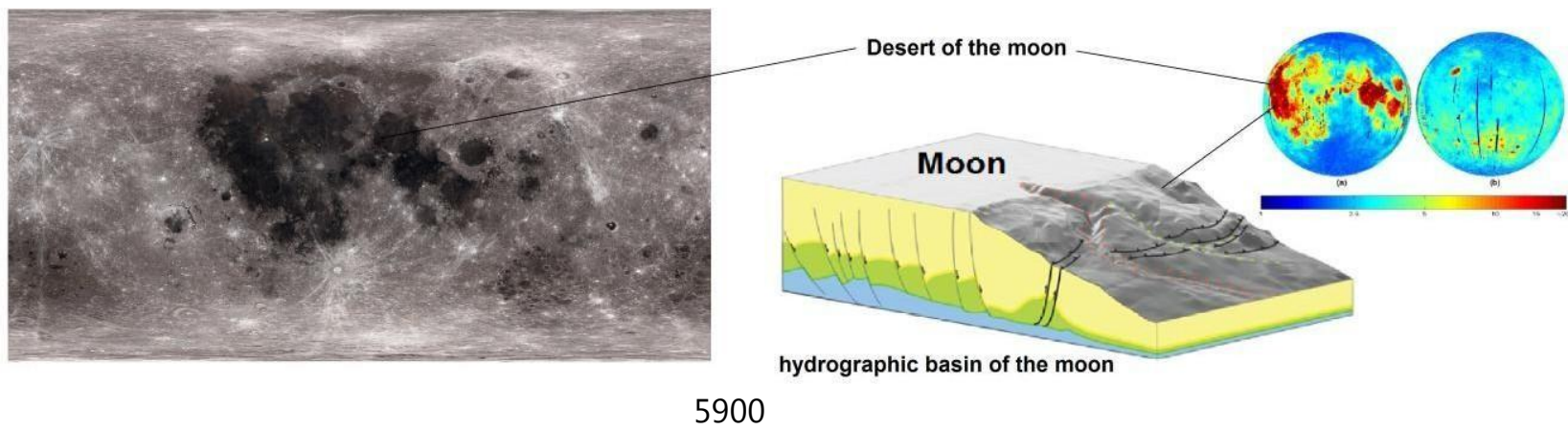




\section{The impact craters from the fall of space debris on the moon are generated by the attraction of the space's gravity field of the moon, and also, then collision with the lunar surface}

All the space debris and meteors that cross the space gravity field of the moon are drawn by the moon and collide on the surface of the moon producing numerous impact craters.

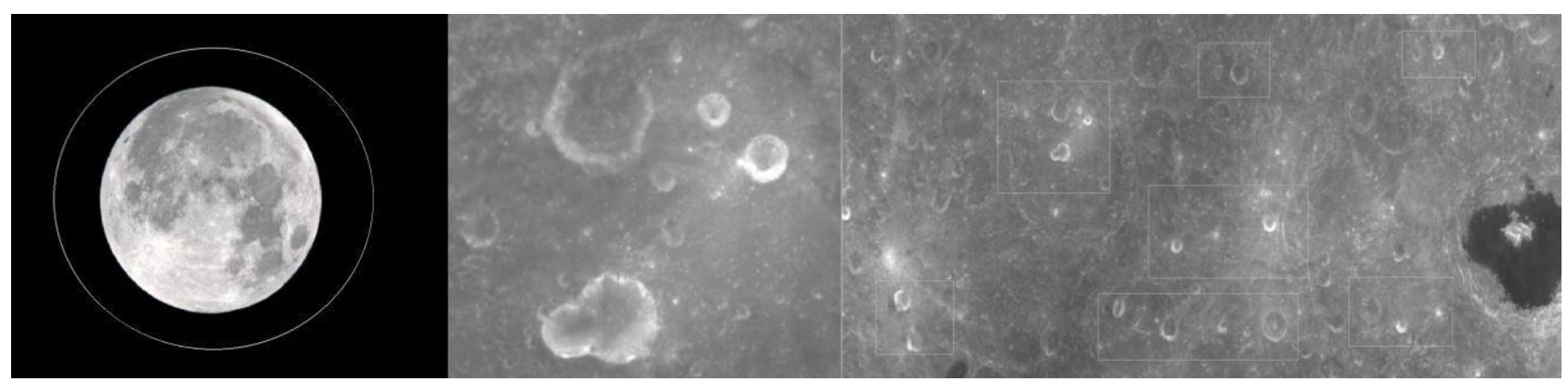

\section{The moon dimension}

The sphere of the moon has a diameter of 3,475 km, however, the sphere of the Earth has a diameter of $12,756 \mathrm{~km}$. So the mass of the sphere of the Earth is 81 times greater than the mass of the sphere of the moon. The diameter of the moon is about $1 / 4$ of the diameter of the Earth.

In this way, we create the first law of the spatial dimension: The mass of the smallest space sphere is always formed as a liquid bubble within the nuclear mass nucleus of the other, much larger spatial sphere.

We still create the second law of the spatial dimension: The mass of the smallest space sphere is expelled from within the nucleus of the nuclear mass of the much larger spatial sphere by the propulsion of nuclear energy from orbital repulsion.

We still create the third law of the spatial dimension: the mass of the smaller space sphere that does not form in the nucleus of the nuclear mass of the largest space sphere is not expelled into space and the bubble collapses because of the force of gravity of the largest space sphere. This space sphere does not have propulsion and sufficient energy level of orbital repulsion.

We still create the fourth law of spatial dimension: The mass of the space sphere of the dwarf planet dimension has no propulsion and energy level of orbital repulsion, to leave the field of the sun's spatial gravity. 


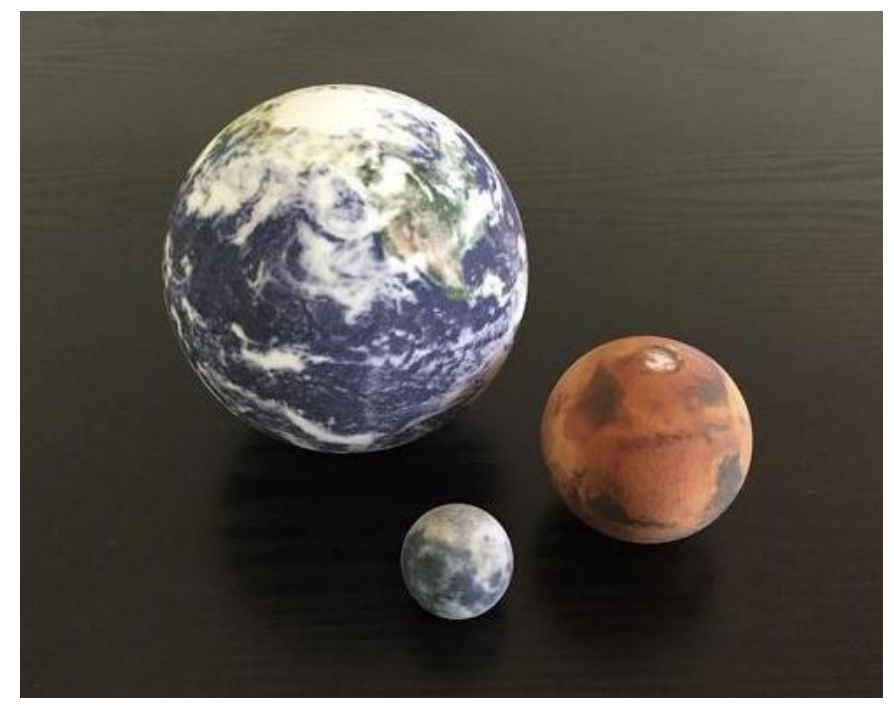

From the images of the celestial bodies, we discover that Mars with the diameter of $6,792 \mathrm{~km}$ and the moon with a diameter of $3,475 \mathrm{~km}$, has underground water basins, but the surface is a deserts of water, due to the insufficiency of the level of the underground gravity.

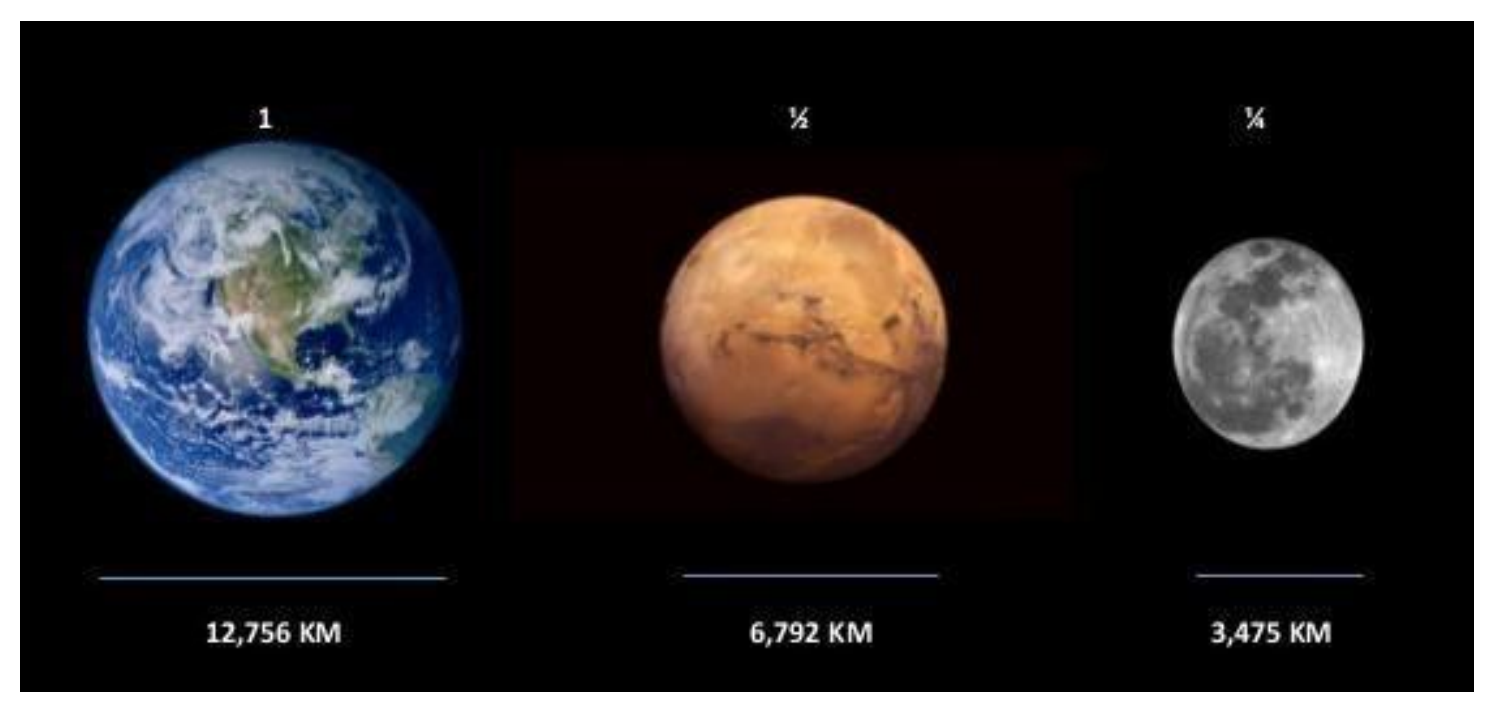

\section{Geography of the lunar pole}

The lunar relief and craters of the lunar pole are geological formations of natural rock, conceived in the relief by solidifying the liquid mass of the moon sphere. 


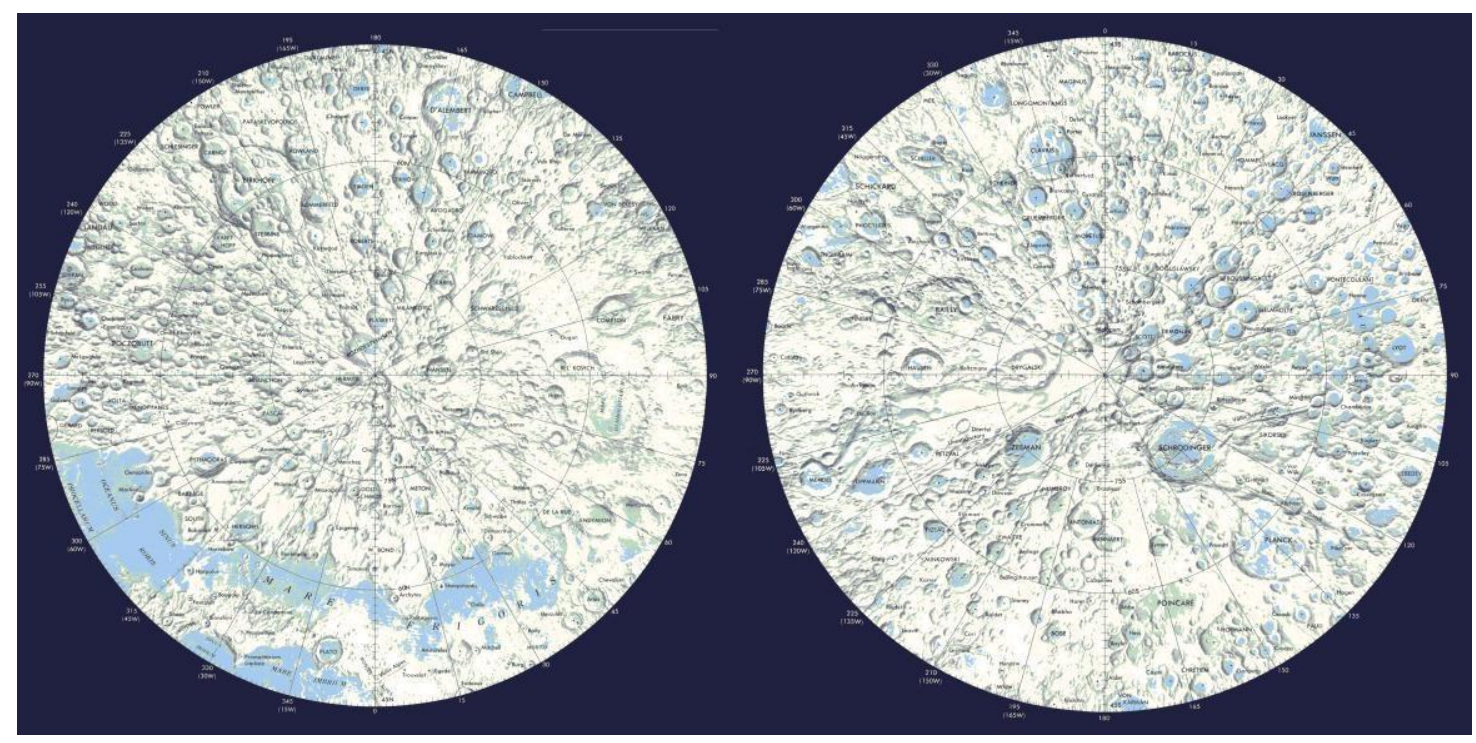

\section{The depressions of lunar equatorial geography}

The depressions of equatorial geography date back to the time of the primitive moon which had several rivers and some seas of water.

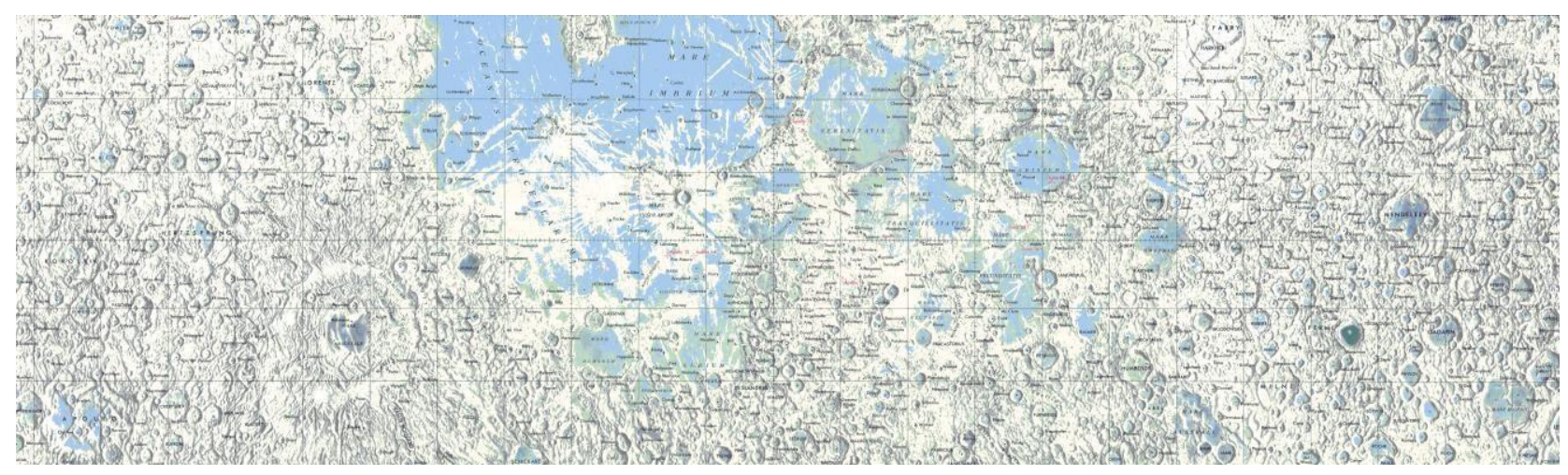

\section{The loss of energy of the nucleus of the primitive moon}

The loss of energy of the gravity of the primitive moon's nucleus transformed the moon into a desert with groundwater. 


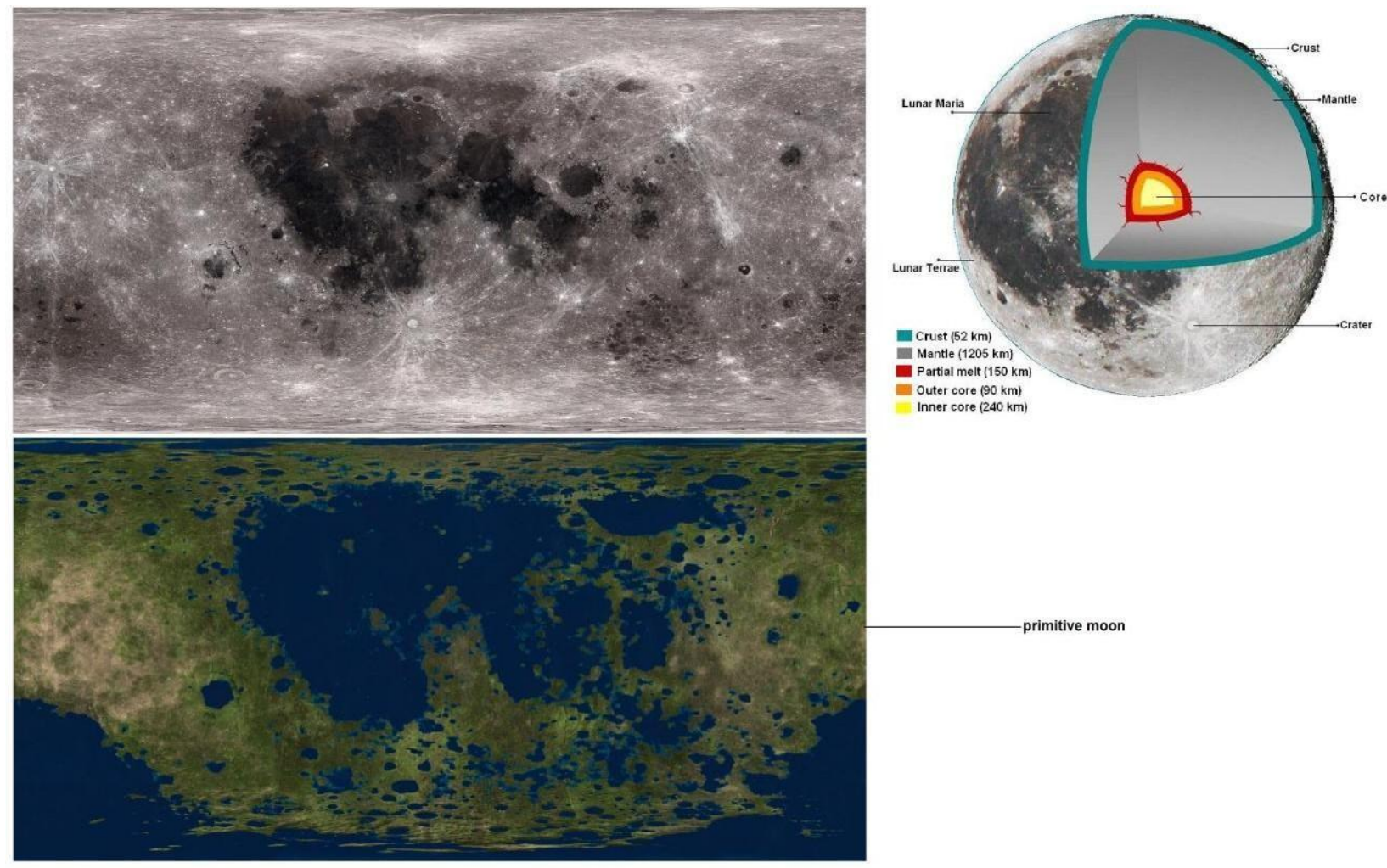

The loss of gravitational energy from the primitive moon nucleus produced a reduction in the kinetic energy of the moon's spin speed

The moon stopped the lunar rotation motion because of the loss of kinetic energy level caused by the loss of energy level of the gravity field of the moon's primitive nucleus.

The loss of gravitational energy from the moon's primitive nucleus produced desertification of the water sources and moon seas

Because of the dimension of the lunar diameter of $3,475 \mathrm{~km}$. The moon had a short plane as an existential biome of life variations. The small diameter spatial spheres have little concentration of nuclear energy of gravity.

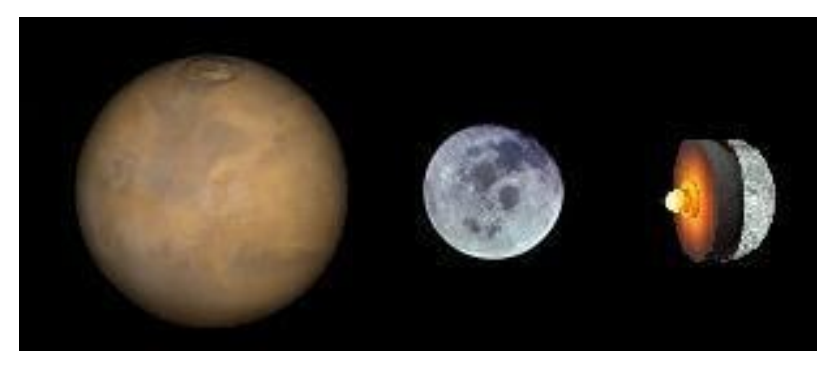

The reduction in the kinetic energy of the rotation speed of the primitive moon produced the loss of the lunar atmosphere

In the case of the existence of a lunar atmosphere in the primitive time. The reduction in the kinetic energy of the rotation speed of the primitive moon produced a slow speed in the lunar rotation and the loss of the 
lunar atmosphere.

\section{A large loss of gravitational energy from the nucleus of the primitive moon produced the kinetic stop of the lunar rotation motion}

As in the ancient legend of the battle in which the moon stopped on the valley of Aijalom. Then, in fact, the rotational stop of the axis of the moon could have happened by the loss of level of the field of lunar gravity.

\section{The loss of energy and the solidification of the moon's nucleus}

The nucleus of the moon by the enormous loss of energy in the form of heat and kinetic energy went from the liquid state to the solid state and then the rotation of the moon stopped.

\section{The loss of orbital repulsion energy from the solidified nucleus of the moon produces the super moon phenomenon}

The super moon is a test phenomenon of the orbital period that demonstrates the loss of the orbital repulsion energy of the moon.

\section{A new energy loss of orbital repulsion of the solidified nucleus of the moon, produces the collision of the moon with the planet}

If no orbital engineering is urgently developed to re-balance within the lunar celestial body the physical ratio of the total mass of the moon with the mass of orbital repulsion energy of the moon. Then the moon will lose more and more orbital sustentation energy, and it will lose the orbital sustentation balance of the orbital energy field until the collision with the planet Earth.

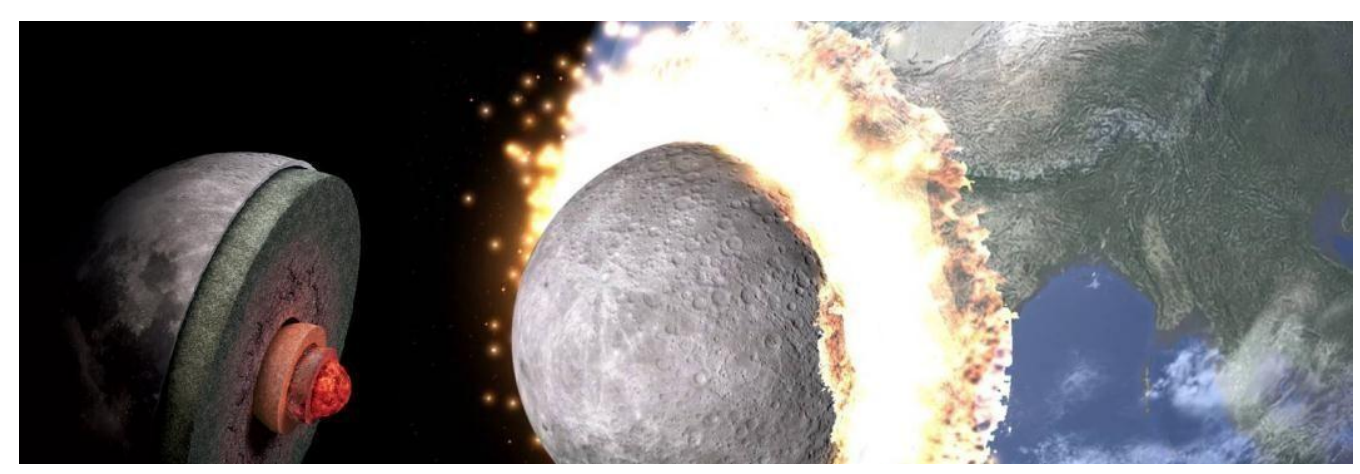

\section{Lunar mirror created in 1971, Apollo 15 mission}

The lunar mirror created by Apollo 15 in 1971 was not developed to measure the phenomenon of the super moon, so the design of 71 , lacks maintenance, lunar dust cleanliness, geographic precision and dimension of the space project. 


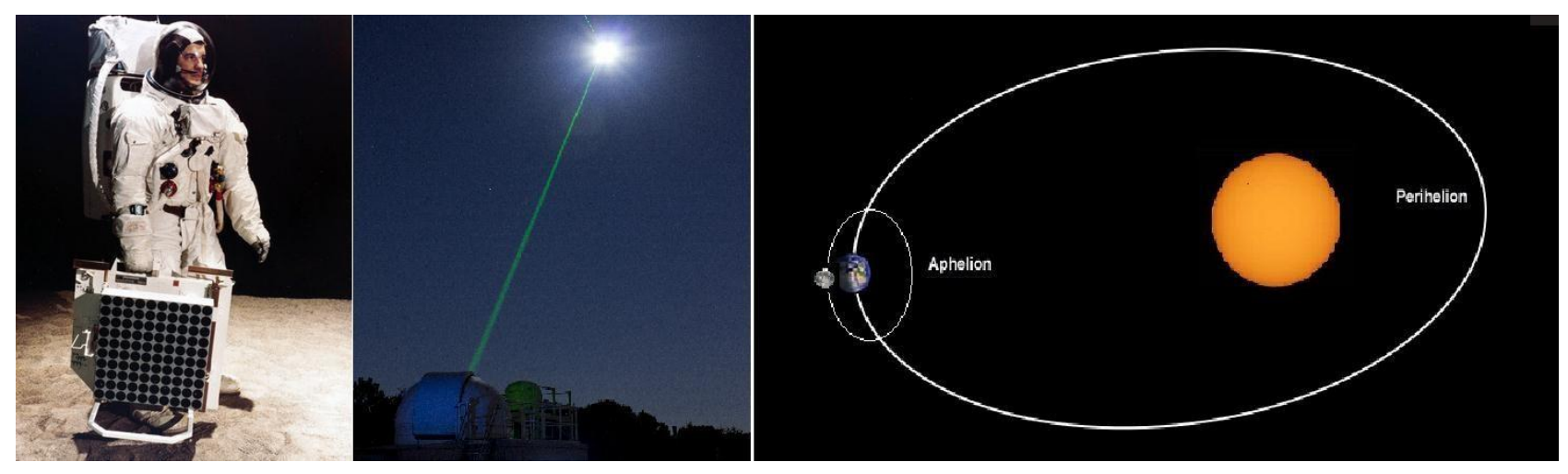

\section{Conclusion}

We conclude that the water of the moon is deposited in underground basins.

We conclude that the impact craters of the fall of space debris on the moon are generated by the mass attraction by the space gravity field.

We conclude that the mass of the sphere of the Earth is 81 times greater than the mass of the sphere of the moon.

We conclude that the space sphere that does not have enough orbital repulsion energy are not formed as planets because they do not pass into space because of the gravity field of the celestial star.

We conclude that the space sphere that does not have enough orbital repulsion energy is not formed as moons, because they do not pass into space due to the attraction of the planet's gravity field.

We conclude by the images of the celestial bodies, that Mars with the diameter of $6,792 \mathrm{~km}$ and the moon with a diameter of $3,475 \mathrm{~km}$, has underground water basins, but the surface of the celestial body is a deserts of water.

We conclude that the loss of gravity energy from the moon's nucleus produced a reduction in the kinetic energy of the moon's rotational speed.

We conclude that the moon stopped the lunar rotation motion because of the loss of the kinetic energy level caused by the loss of the energy level of the gravity field of the primitive moon's nucleus.

A redução na energia cinética da velocidade de rotação da lua primitiva produziu uma velocidade lenta na rotação lunar e a perda da atmosfera lunar.

We conclude that the reduction in the kinetic energy of the rotation speed of the primitive moon was the main consequence of the solidification of the mass of the lunar nucleus.

We conclude that the disintegration of the lunar atmosphere was the main consequence caused by the reduction of the speed of the rotation of the primitive moon.

We conclude that the loss of orbital repulsion energy from the solidified nucleus of the moon produces the super-moon phenomenon.

We conclude that the moon's nucleus went from the liquid state to the solid state and then the moon's rotation stopped.

We conclude that the super moon is a test phenomenon of the orbital period that demonstrates the loss of 
the orbital repulsion energy of the moon.

We conclude that in the future the moon will lose more and more orbital sustention energy, and will lose the orbital sustention balance of the orbital energy field.

We conclude that the lunar mirror created by the Apollo 15 in 1971, lacks maintenance, cleaning of the lunar dust, geographical precision on Earth.

\section{Annex 3 - The Fall of the Moon of Jupiter}

\section{The myths of the gaseous planet}

The myths of the gaseous planet, Jovian planet or giant gas planet would be some medieval myths of large planet that would be composed mainly of gas (Hydrogen, Helium, Methane). By the myth there are four of these planets in the solar system: Jupiter, Saturn, Uranus and Neptune.

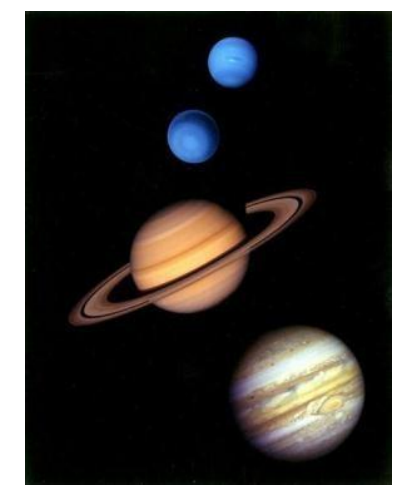

In fact, there can not exist a giant gas planet because it violates the principle of isotropy and the principle of homogeneity of the matter of formation of the celestial bodies.

In fact, there can not exist a giant gas planet because it violates the rule and principle of the geological nuclear masses of the orbit, which produce the orbital energy field between the nucleus of the moons with its planet and between the nucleus of the planets with its star, that say:

Thus, by the new theory, every celestial body has an orbital nuclear superconductor which is the nucleus of the celestial body, which produces the field of orbital energy and also produces the field of magnetism, and the gravity field of the celestial body.

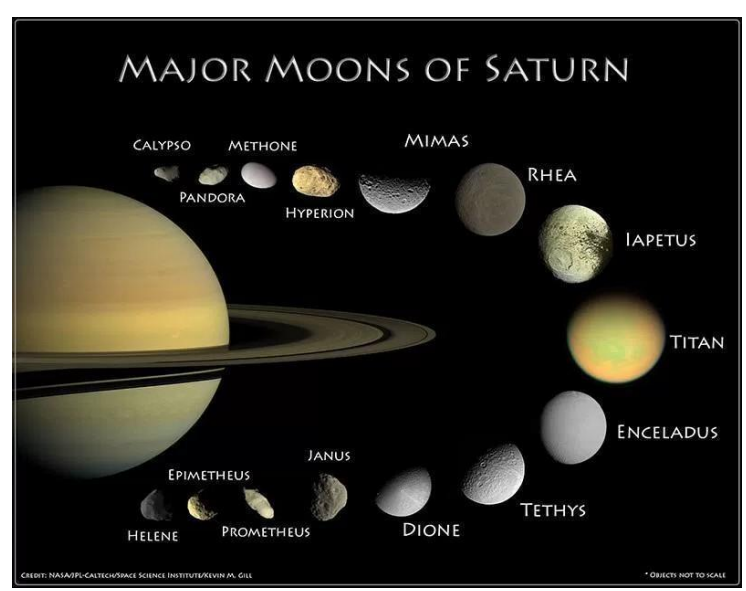


The myth of the giant gaseous planets also hurts the basic principle of homogeneity of the universe, which shows that all planets are made up of the same matter, and with the same properties. That is, what we can prove is that the Earth, Mars and Venus are solid planets, so that we can conclude that all other planets in the solar system were formed identically, regardless of their size.

If there were a gigantic gaseous planet, they would not have the same orbital dynamics as the other planets in the solar system. If there were a gigantic gaseous planet, they could not have solid moons.

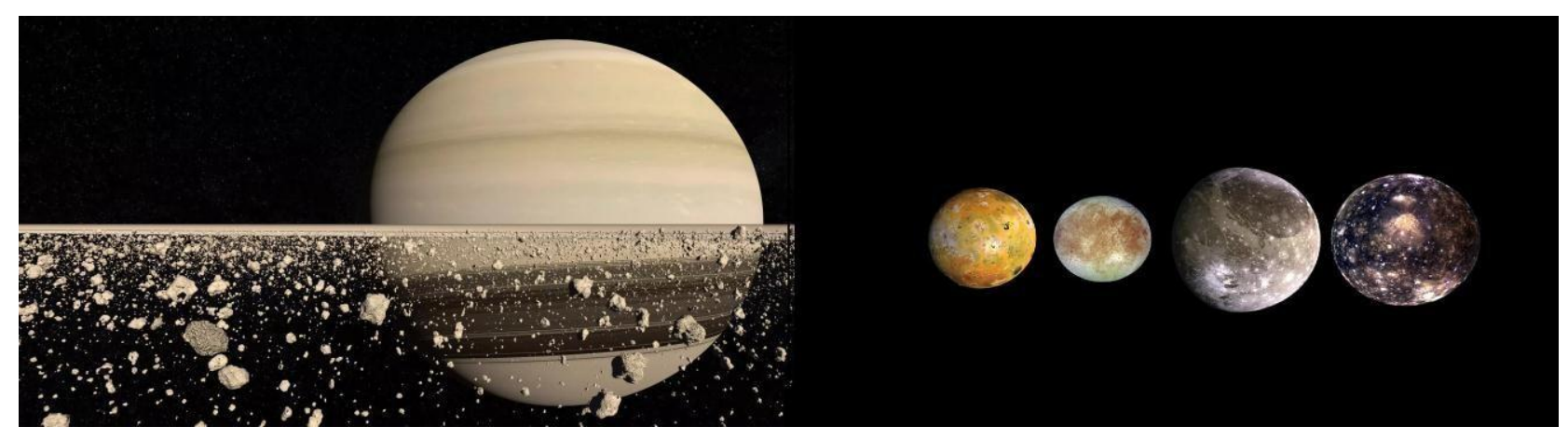

A gaseous planet could not have solid moons.

A gaseous planet could not have billions of solid mass fragments orbiting Saturn's rings.

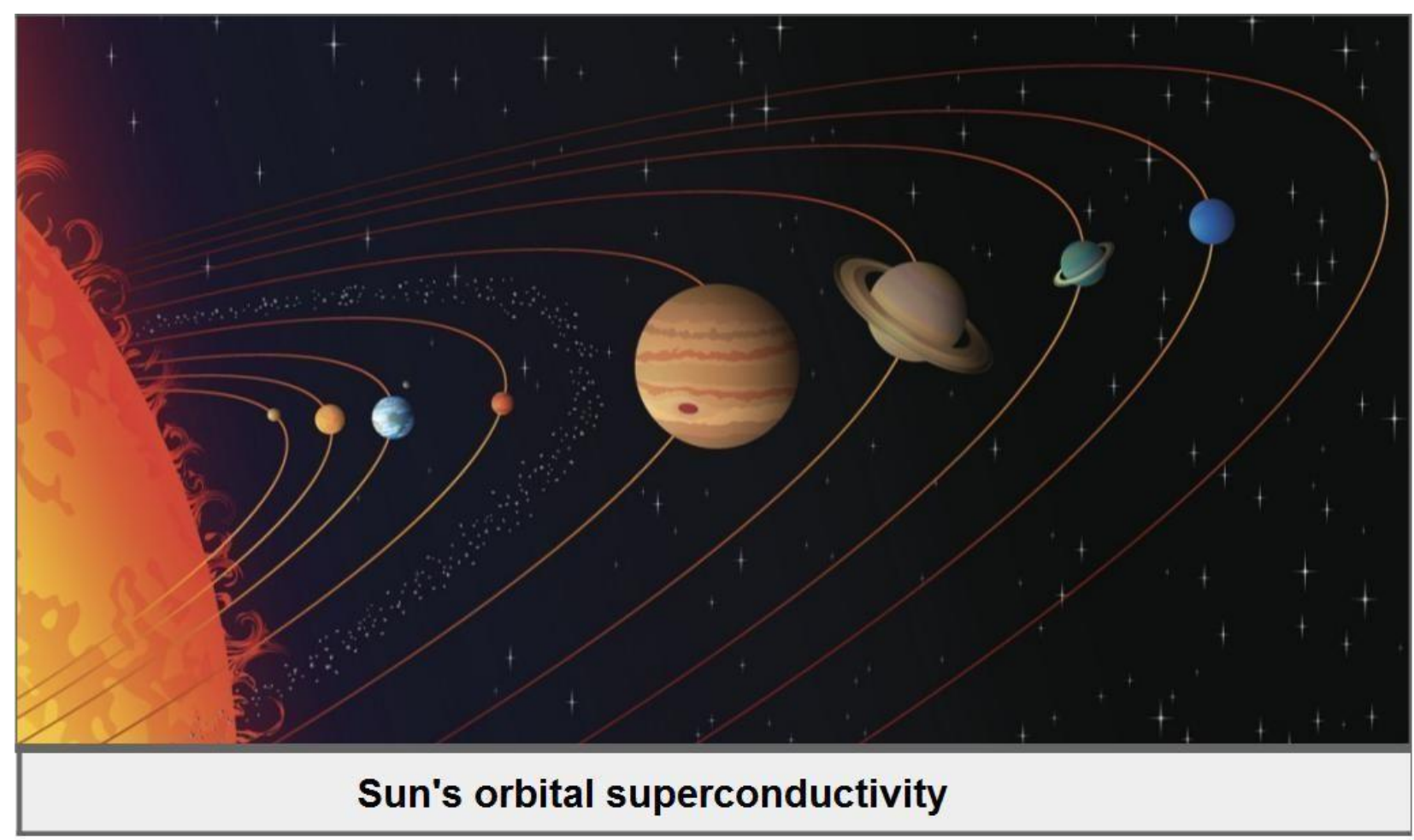

The creation of Jupiter and the creation of the five moons of Jupiter

All the planets were created from the same orbital nuclear material and by the same principles of nuclear physics. 

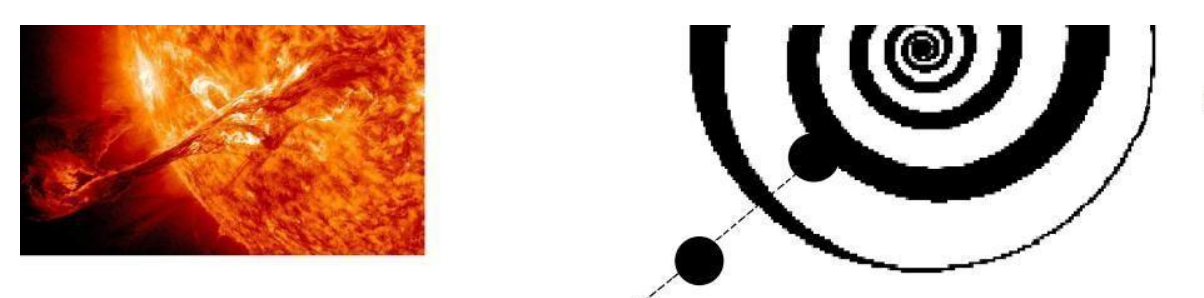

Nucleus of the Sun and the exit of Jupiter

Sun in the liquid state and Jupiter in the liquid state
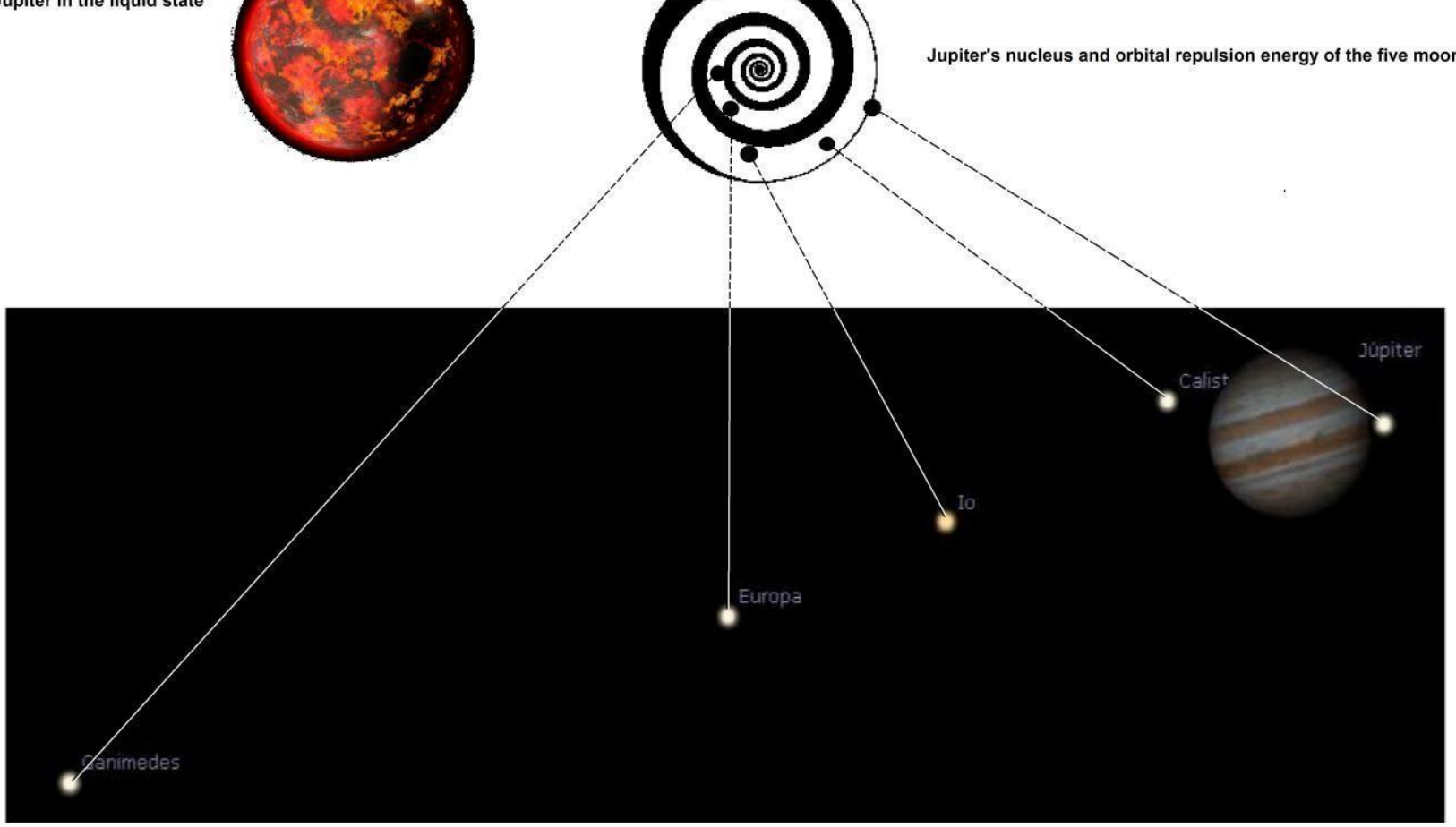

\section{The fall of Jupiter's fifth moon}

Long before the men of the planet Earth of the 17th century, know the images of the telescope of the great inventor Galileo Galilei. The fifth moon had already fallen on Jupiter. Then, at that time, Jupiter had only five natural moons.

Because it originated naturally, far from the center of Jupiter's core. Jupiter's fifth moon has less orbital repulsion energy, and prematurely fell on Jupiter, before the other orbiting moons.

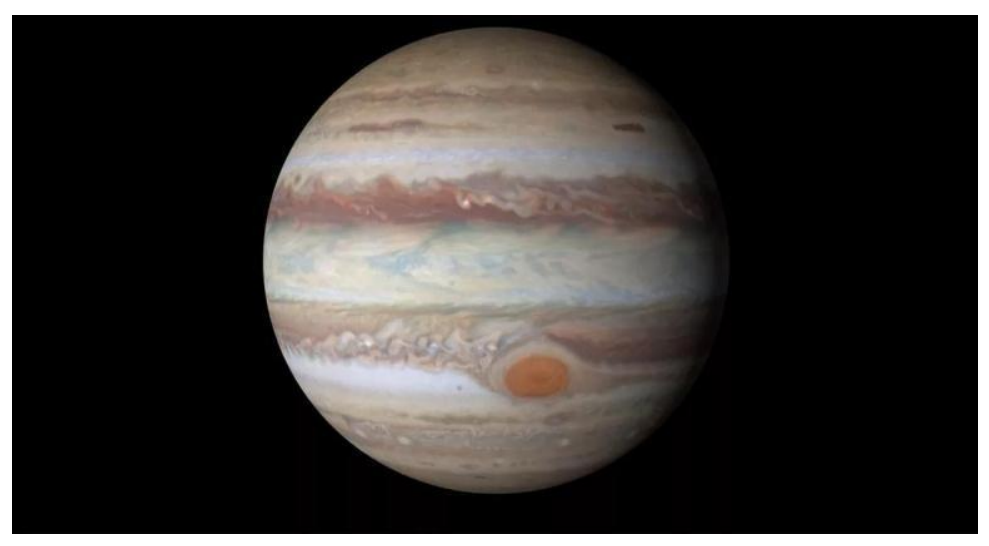


The fall of Jupiter's fifth moon has changed the landscape of the solar system.

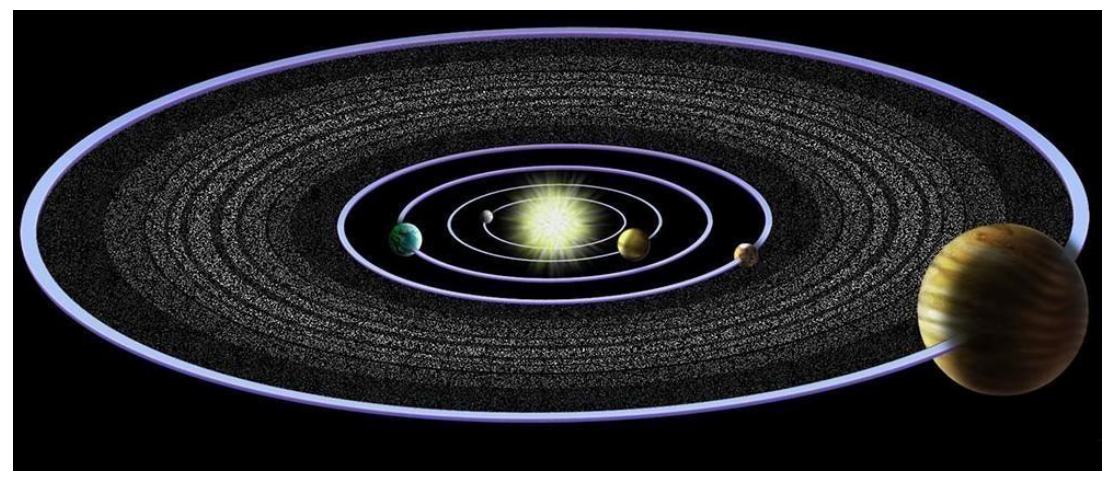

Notice that the asteroids of Troy are anchored in the orbit of Jupiter.

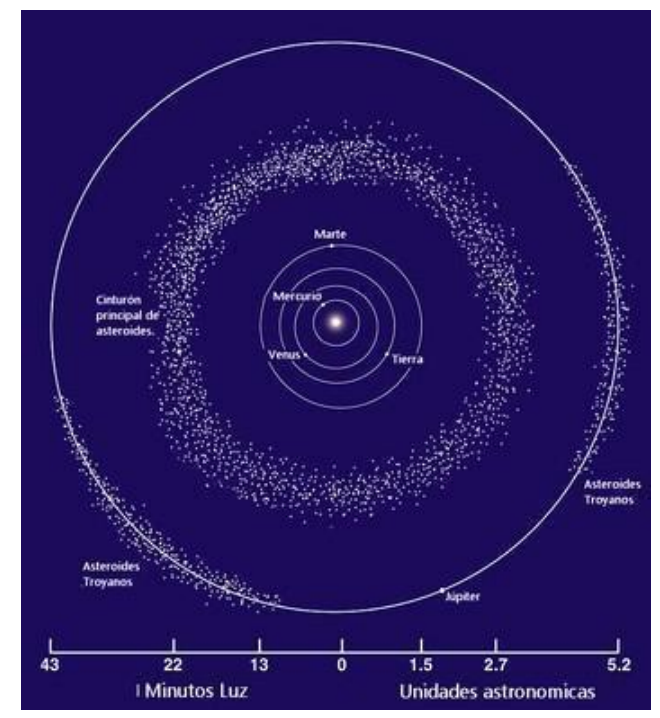

Already in another orbit between Mars and Jupiter we noticed an asteroid belt with enormous volume of asteroids.

\section{The abyss of Jupiter is hot and immense gravitational hurricane}

The Jupiter's moon solidified the nucleus, lost the orbital support energy, and then the moon cooled and approached irregularly of Jupiter, entering an orbit of immediate collision.

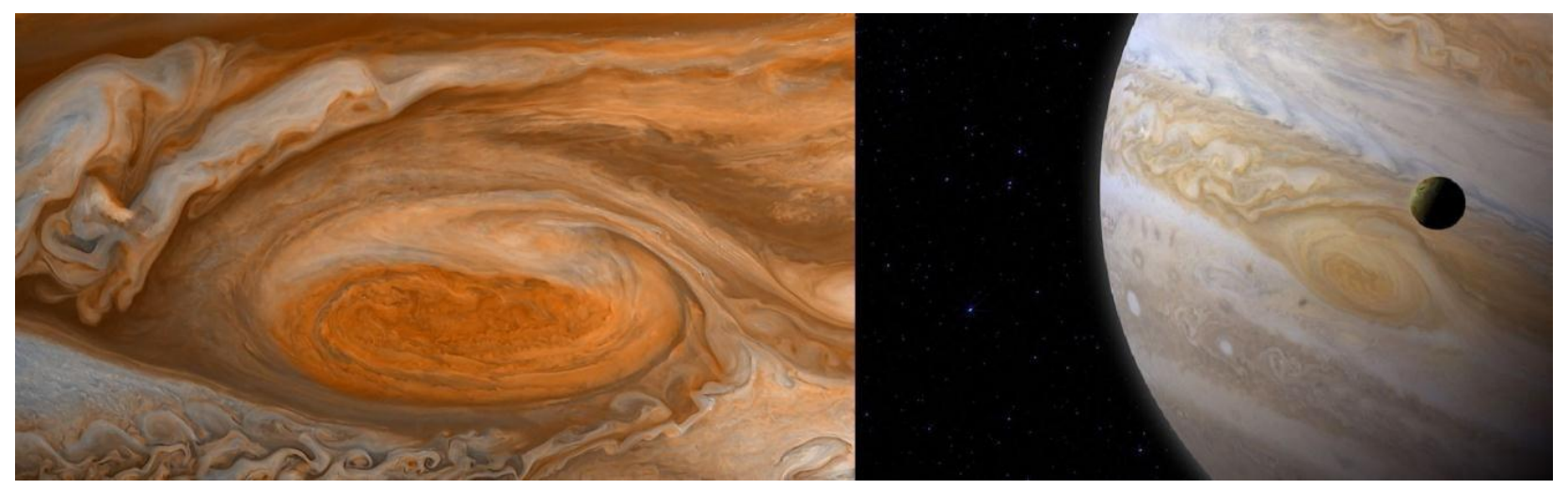


The fall of Jupiter's moon entered the depths of the subterranean layers of Jupiter, and produced a super gigantic abyss in Jupiter.

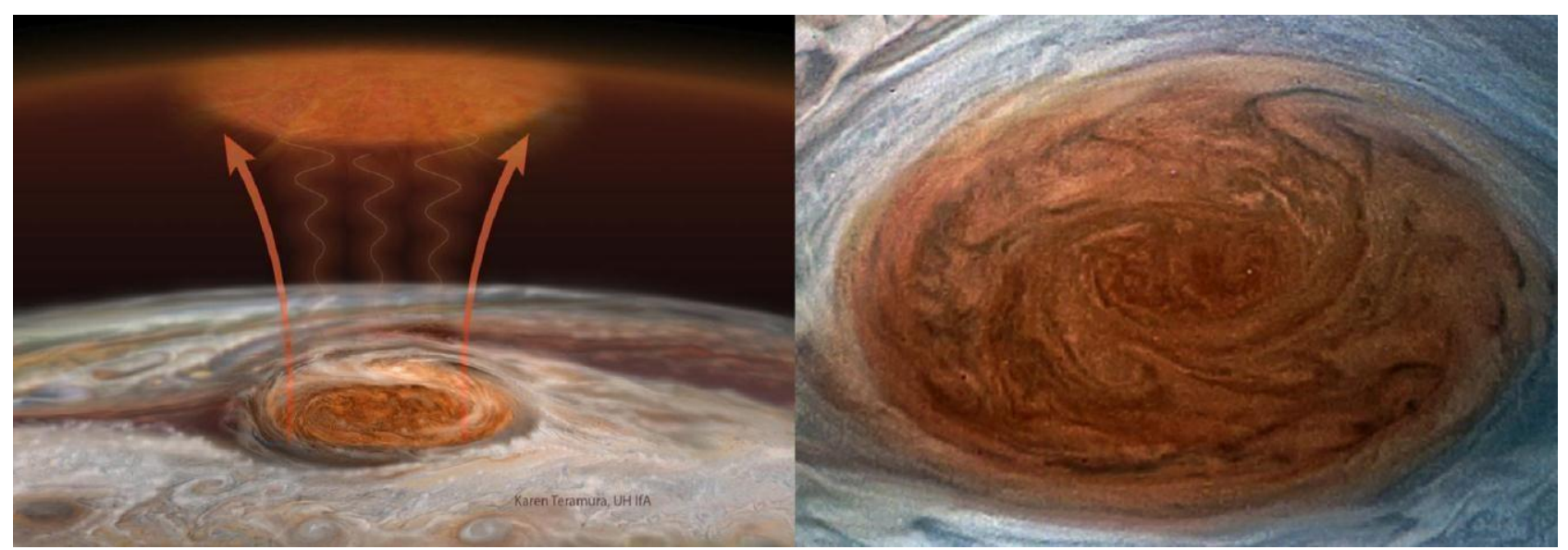

Then, by destroying Jupiter's moon by the impact of the collision, the Jupiter Moon's core was trapped in the abyss of Jupiter, and the gravity geological nuclear mass produced a monstrous super-gravitational hurricane with continuous velocity creating winds over $620 \mathrm{~km} / \mathrm{h}$.

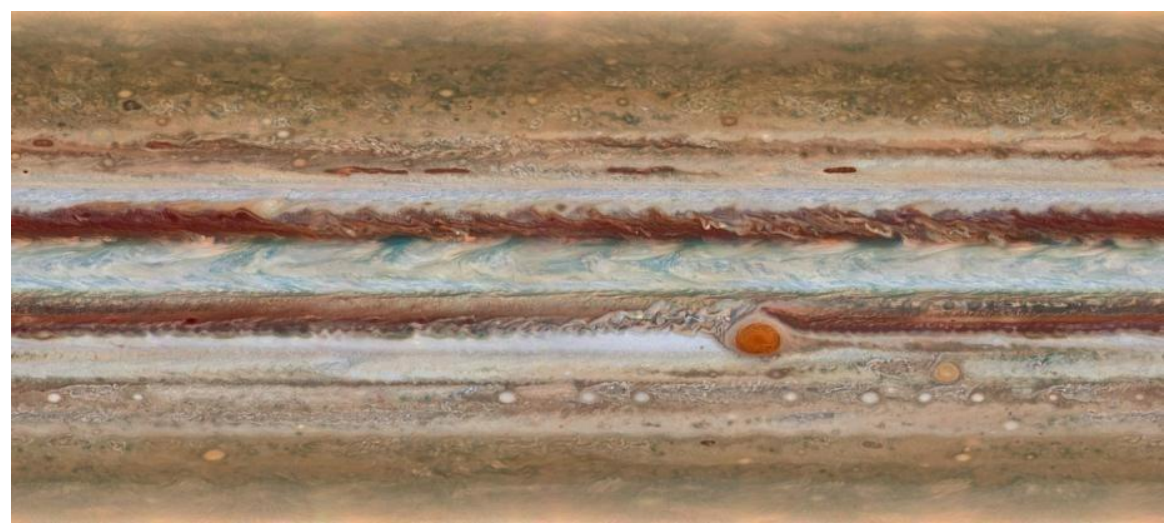

The fall of Jupiter's moon is responsible for the super-planet's high temperature. The huge red hurricane is twice as big as Earth and is $600^{\circ} \mathrm{C}$ warmer than the rest of the planet. It pumps heat from the interior of the planet to waves that run through the entire circumference of Jupiter.

\section{The creation of asteroid belts}

In the fall of the moon of Jupiter there was the creation of the belt of asteroids and the asteroids called Trojans, that are to the right and left side of Jupiter.

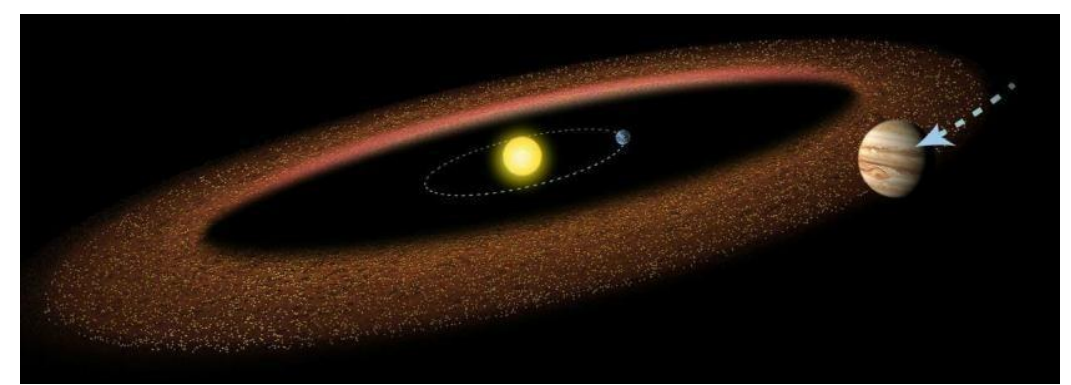


In 1868 a hundred asteroids were known, and in 1891 the discovery of astrophotography by Max Wolf accelerated this rhythm even more.

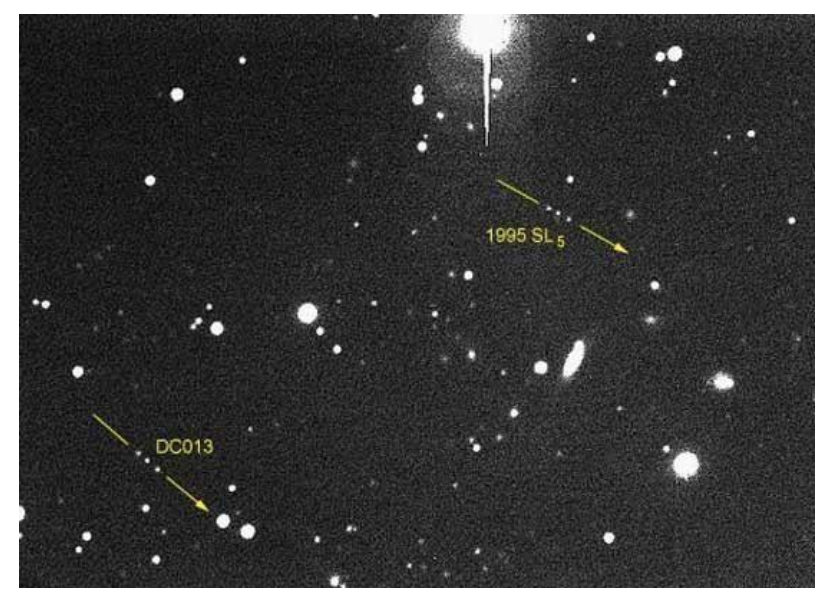

In 2010 the number of asteroids is about 500000.

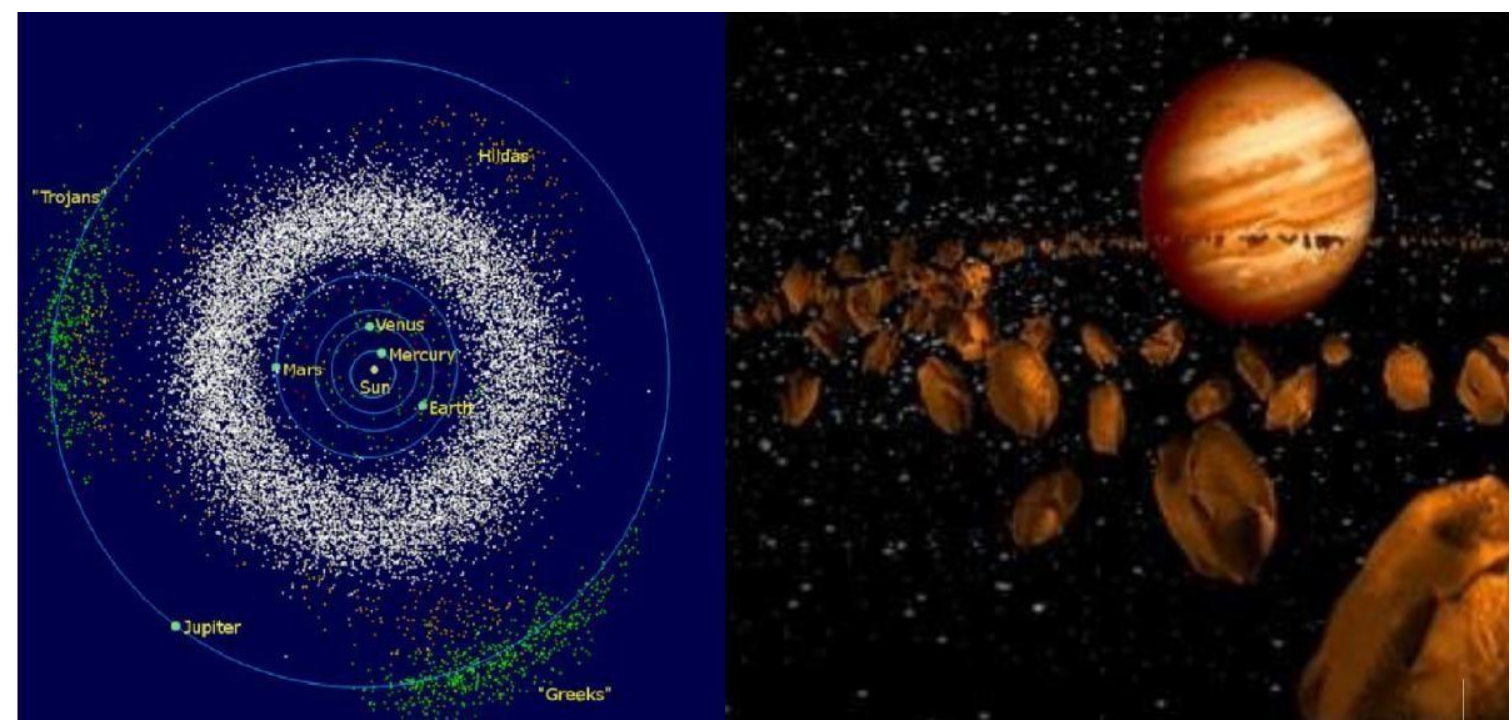

\section{Conclusion}

We conclude that without the physical model of the geological energy of gravity, the super planets Jupiter, Saturn, Uranus and Neptune would not have the rotation movement on the axis, and therefore, the atmosphere of the super planets would have been dissolved by the property of the space of formation of bubbles in space.

We conclude that the model of the giant gas planet is a medieval myth.

We conclude that Galileo Galilei when he first observed Jupiter can not understand the origin of the Jupiter stain. For at that time without electric light, man did not understand the laws of gravity, and therefore he did not understand the phenomena of physics and astronomy.

We conclude that the myth of giant gaseous planets also hurts the basic principle of homogeneity of the universe, which shows that all planets are composed of the same matter and with the same properties.

We conclude that we can prove that Earth, Mars and Venus are solid planets, so we can conclude that all 
other planets in the solar system were formed identically, regardless of their size.

We concluded that if there were a gigantic gaseous planet, they could not have solid moons.

We conclude that all planets were created from the same orbital nuclear material and by the same principles of nuclear physics.

We conclude that the myth of the gaseous giant planet has no foundation in physics.

We concluded that long before the men of the planet Earth of the 17th century, to know the images of the telescope of the great inventor Galileo Galilei. The fifth moon had already fallen on Jupiter.

We conclude that the fall of Jupiter's moon is a physical phenomenon, which occurred at some date, prior to the fabrication of the Galileo Galilei telescope at the beginning of the 17th century (November 1609).

We conclude that because the fifth moon of Jupiter originated naturally, away from the center of Jupiter's nucleus. Jupiter's fifth moon has less orbital repulsion energy, and fell prematurely on Jupiter, before the other four orbiting moons.

We conclude that the moon of Jupiter entered the depths of the subterranean layers of Jupiter and produced a super gigantic abyss in Jupiter.

We conclude that in the fall of the moon of Jupiter there was the creation of the so-called Trojan asteroids, which are on the right and left side of Jupiter.

\section{Annex 4 - The physical property of the geology}

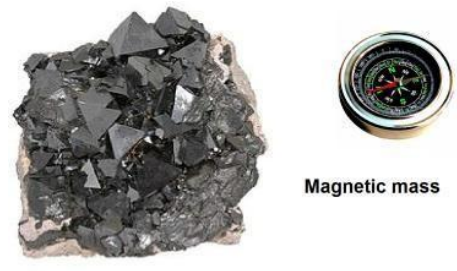

Property of natural geology

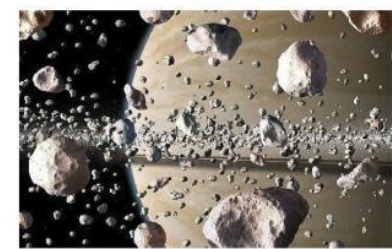

Orbital mass

Property of natural geolog

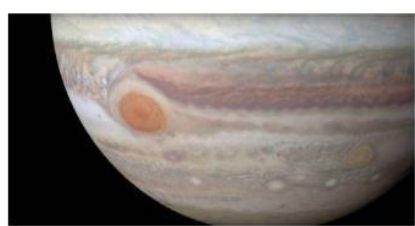

Gravity mass
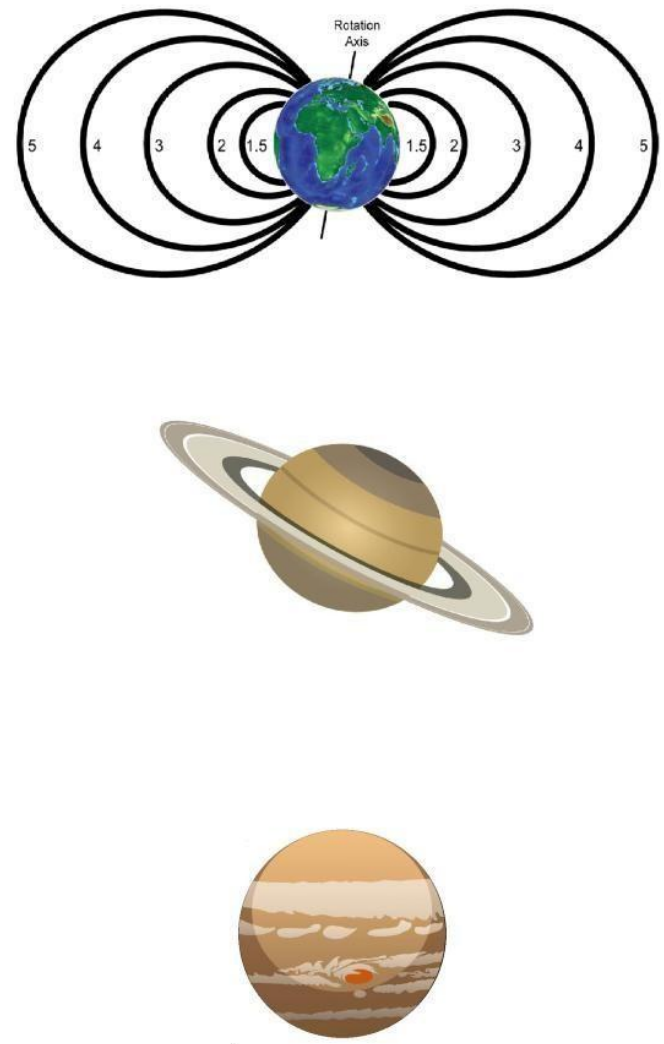

Property of natural geology 
The geology is the great-grandmother of almost all sciences. Man would not exist without geology. History does not exist without geology and without man. Mathematics does not exist without man. Physics does not exist without geology and without man. The geography would not exist without geology. the chemistry would not exist without man. Astronomy would not exist without geology, without physics, and without man.

So unlike nature, only a Mega Special Supercomputer can replicate the subatomic phenomena of geology, physics, and math.

Therefore, we understand that physical and geological property naturally generated the field of magnetic energy; the orbital energy field; the energy field of gravity of the celestial bodies.

Right in the real world, there is no universe of gaseous planets, spell, and magic.

\section{Conclusion}

We conclude that the "Theory of the three fields of space" are well grounded in the nature of geology. 
The creation of the solar system was the most important questioning of science. Then in 1948 the astronomer Georges Lemaitre created the Big Bang theory of the origin of the universe, but based on the scientific fiction that the Universe began from an extremely dense point and that, when it exploded, created the cosmos. Then, in 2018, inventor Eduardo Guimarães made use of the Big Bang theory, but based on the new concept of its creation: the physics of the nuclear universe, through the studies "The Theory of the Nuclear Universe and The Secret of the Supernova", and by the study "Theory of the three fields of space", and by this study, "The lunar problem is the barrier of the future time of the Earth". 